\title{
A metamodel-based Monte Carlo simulation approach for responsive production planning of manufacturing systems
}

\author{
Minqi Li ${ }^{\mathrm{a}}$, Feng Yang, ${ }^{\mathrm{a}, *}$ Reha Uzsoy ${ }^{\mathrm{b}}$, Jie $\mathrm{Xu}^{\mathrm{c}}$ \\ ${ }^{a}$ Industrial and Management Systems Engineering Department, West Virginia University, Morgantown, \\ WV 26505, USA \\ ${ }^{b}$ Edward P. Fitts Department of Industrial 8 Systems Engineering, North Carolina State University, \\ Raleigh, NC 27695, USA \\ ${ }^{c}$ Department of Systems Engineering $\&$ Operations Research, George Mason University, Fairfax, VA \\ 22030, USA
}

\begin{abstract}
Production planning is concerned with finding a release plan of jobs into a manufacturing system so that its actual outputs over time match the customer demand with the least cost. For a given release plan, the system outputs, work in process inventory (WIP) levels and job completions, are non-stationary bivariate time series that interact with time series representing customer demand, resulting in the fulfillment/non-fulfillment of demand and the holding cost of both WIP and finished-goods inventory. The relationship between a release plan and its resulting performance metrics (typically, mean/variance of the total cost and the fill rate) has proven difficult to quantify. This work develops a metamodelbased Monte Carlo simulation (MCS) method to accurately capture the dynamic, stochastic behavior of a manufacturing system, and to allow real-time evaluation of a release plan's performance metrics. This evaluation capability is then embedded in a multi-objective optimization framework to search for near-optimal release plans. The proposed method has been applied to a scaled-down semiconductor fabrication system to demonstrate the quality of the metamodel-based MCS evaluation and the results of plan optimization.
\end{abstract}

Keywords: production planning, metamodeling, non-stationary time series, Monte Carlo simulation, multi-objective optimization

\section{Introduction}

This work is concerned with production planning in manufacturing, which can be defined as the problem of finding a release schedule of jobs into the manufacturing system so that the realized outputs over time satisfy predetermined requirements as close as possible [1]. The planning horizon of production activities usually ranges from several months to two years, and the frequency of planning/replanning is weekly or monthly [2].

*Corresponding author. Tel.: +1 3042939477

Email address: Feng. Yang@mail.wvu.edu (Feng Yang ) 


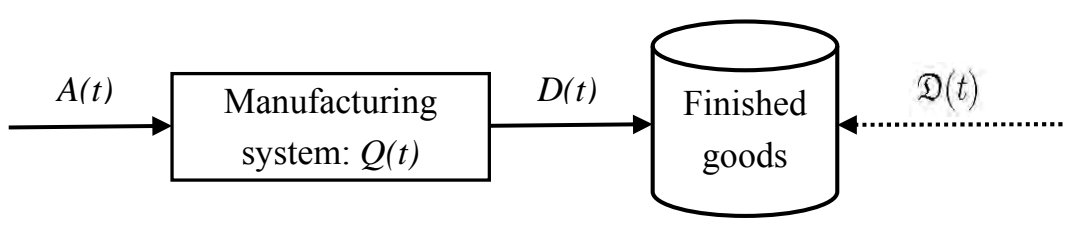

Figure 1: The input-output process of a manufacturing system.

Typically, the planning horizon is divided into a number of discrete periods, and the decision variables represent the quantities of work of different types released into the system in each period. The performance metrics to be optimized usually include (i) the total cost (or profit), which may consist of the holding cost for finished goods (FG) and work in process (WIP) inventories, production costs and backordering costs and (ii) the fill rate, defined as the percentage of immediately satisfied demand.

Optimizing the performance metrics with respect to (w.r.t.) the release plan is challenging because it is notoriously difficult to quantify the relationships between the performance metrics and the input decisions. A manufacturing system is subject to inherent uncertainties such as probabilistic processing times, machine failures, etc., leading to complicated inputoutput relationships as discussed in Section 3. We shall focus on three principal time series: $A(t)$, the number of jobs released for processing during period $t, Q(t)$, the number of jobs (i.e., WIP) in the system at the start of period $t$, and $D(t)$ the number of completed jobs departing from the system during period $t$. Figure 1 illustrates the input-output process of a manufacturing system. The release process $A(t)$, which will usually vary over time, is determined by the decision variables (the release plan). Given $A(t), Q(t)$ and $D(t)$ are non-stationary time series describing the system's outputs over time, whose evolution also depends on the initial status of the system. The realized performance metrics depend on $Q(t), D(t)$, and the customer demand $\mathfrak{D}(t)$; the WIP holding cost incurred is determined by $Q(t)$; and the FG holding cost and the fill rate by the departure process $D(t)$ and the customer demand $\mathfrak{D}(t)$. In practice, demand is generally a non-stationary time series, and is specified through forecasting efforts exogenous to production planning.

Despite extensive research, it remains a challenge to adequately quantify the relationship between the performance metrics and the release schedule due to the complex interactions between the non-stationary time series $A(t), Q(t), D(t)$, and $\mathfrak{D}(t)$. To address this difficulty, this paper develops a metamodel-based Monte Carlo simulation (MCS) method with the following features. First, for a given release plan, it enables the evaluation of the probabilistic measures of system performance including expectations (e.g., the mean cost), variances (e.g., the variance of the cost) and probabilities of interest (e.g., the fill rate). Second, it is able to accommodate a wide variety of demand patterns. Third, it allows rapid evaluation of a candidate plan in terms of its performance metrics, and permits timely plan optimization.

The remainder of this paper is organized as follows. Section 2 provides a review of 
the related literature. Section 3 gives an overview of the metamodel-based MCS method for responsive production planning. Section 4 details the input-output metamodeling of a manufacturing system, and the metamodel-based MCS is discussed in Section 5 . Section 6 formulates the multi-objective optimization problem for production planning, and presents the optimization scheme that uses the MCS method to quickly evaluate each candidate plan. In Section 7, the plan optimization approach is applied on a scaled-down semiconductor fabrication system. A brief summary is given in Section 8 .

\section{Literature Review}

Production planning problems have been addressed by several different streams of research, each emphasizing certain aspects of the problem, and thus different mathematical models. A strong case can be made [3] that none of these formulations addresses the problem faced in industry in its full complexity and generality. Hence in practice the production planning function will often combine several different mathematical models. In addition, it is possible to formulate the production planning problem in different ways depending on the time frame covered and the level of information aggregation involved [4, 5]. In this paper we shall focus on a limited formulation, that of how to release work into a production system over time in order to match its output with demand in some optimal or near-optimal manner.

A central issue in matching production output to demand arises from the presence of substantial cycle times in most production systems. The cycle time is defined as the time elapsing between a unit of work (a job) being released into a production system and its emergence from the system as a finished product. In a practical production system the cycle time of any given job is a random variable whose distribution potentially depends on all uncertainties arising in the production environment, such as behavior of human decision makers, process times, machine failures, and so on. Queueing models ([6, 7]), simulation models (e.g., [8, 9]) and empirical observation are all in agreement that the distribution of the cycle time will also depend on the average utilization of the production resources. This creates a central difficulty for production planning systems: in order to match output to demand, they need to consider cycle times; but the distribution of the cycle time is determined to a considerable degree by the resource utilization, which, in turn, is determined by the release decisions made by the planning system. Hence cycle times are endogenous to the release decisions made by the planning system. However, cycle times are determined in practice by complex interactions between several complex stochastic processes evolving over time, such as the pattern of releases into the system, customer demand, machine failures, and the arrival of jobs at machines within the system over time. The difficulty of a comprehensive analytical treatment of these interactions in their entirety constitutes the central difficulty faced by production planning. 
Mathematical programming models have focused on the allocation of limited resource capacity among different products over time. Much of this work assumes all inputs are deterministic, leading to formulations as linear or mixed integer programs [10 14]. Most of these models, as well as the widely used Material Requirements Planning (MRP) approach [15 17] treat cycle times as an exogenous parameter independent of resource utilization. These models ignore the stochastic nature of the problem, requiring enhancements to their solutions to be useful in practice, and also ignore the relation between release decisions and cycle times. However, deterministic mathematical programming models have been used extensively in industry as the basis for successful planning systems [18, 19]. These deterministic models have been extended in several ways to incorporate uncertainty in both production and demand. Several authors [20 23] have proposed scenario-based stochastic programming models. The main difficulty with this approach is the extremely rapid growth in the size of the models as the number of decision epochs and random variables increases. A variety of robust optimization approaches have been proposed, in which one seeks a production plan that will provide a satisfactory solution over a restricted set of uncertain outcomes [24, 25]. Yet another approach has been the use of chance constraints, where constraints may be violated with a specified probability [26, 27]. These techniques tend to be computationally less demanding than stochastic programming, but also represent uncertainty and its consequences in different ways. Aouam and Uzsoy [28, 29] compare a number of these models in the context of a very simple single-stage production-inventory system under stochastic demand, and find that they need to be parameterized with care to yield desirable results.

Another extension of mathematical programming models has been in the direction of explicitly representing the dependence between cycle times and planning decisions, making cycle times endogenous to the planning models [30, 31]. One such approach, which is closely related to this work, is the use of nonlinear clearing functions that represent the expected output of a production resource during a planning period as a function of some measure of its planned workload during that period. The planned workload, is usually computed from a set of state variables such as the total amount of work available to the resource in the period or the average work in process (WIP) level during the period. The clearing function is usually assumed to be, and can in many cases be shown to be, a concave nondecreasing function of the state variables which admits of piecewise linearization, yielding tractable optimization models. Simple clearing functions can be derived from classical steady state queueing models (e.g., [32, 33]) or transient queueing models [14, 34, 35]. In general, the form of the clearing function will change from period to period based on the values of the state variables used to estimate it. However most models using clearing functions have assumed a time-stationary function representing the expected performance of the system over an appropriate range of operating conditions. Models using clearing functions have shown considerable promise in extensive computational experiments [33, 36 38]. The work in this paper can be viewed as a generalization of the clearing functions employed to date, 
extending the number and nature of the state variables considered and explicitly modeling the evolution of the clearing function over time based on the evolution of the underlying state variables.

While these approaches may still be sufficient in many cases to provide important insights, detailed discrete-event simulation (DES) models appear to be the only methodology that permits the detailed modelling of complex stochastic systems and their interactions that typify production systems. However, DES comes with its own set of disadvantages, namely the increasing computational burden as the system under study is modeled in more detail. These computational requirements are further exacerbated by the need for multiple replications to ensure an acceptable degree of statistical precision in the results.

For these reasons simulation optimization has not been extensively applied to production planning problems of the type addressed in this paper. The natural formulation of the problem would be to consider the releases in each period as the decision variables. However, for problems with many time periods and products, this results in a very large decision space. Hasan and Spearman [39] consider the problem of determining the optimal release times for a fixed sequence of work using a simulation-optimization approach based on infinitesimal perturbation analysis. This approach is further developed by Homem de Mello et al. [40]. Liu et al. [41] use a simulation-optimization approach based on a genetic algorithm to obtain optimal release schedules for the simplified wafer fabrication facility considered in this paper, and compare the results to those from a an optimization model using clearing functions. They find that the simulation-optimization approach yields better solutions than the clearing function model at the cost of significantly higher computation time. Kacar and Uzsoy [42] confirm this finding. A number of authors have used simulation optimization techniques to estimate the values of parameters used in more traditional production planning models. Gansterer et al. [43] use simulation optimization to determine the optimal values of planned lead times, safety stock levels and lot sizes in an MRP environment. Albey and Uzsoy [44] similarly use simulation optimization to determine values of planned lead times for use in linear programming models. Kacar and Uzsoy [42] use simulation optimization to optimize the parameters of clearing functions which are then used in an optimization model. In general, the computational time requirements of simulation optimization based on detailed DES models renders their direct use for optimizing release plans in large systems impractical, although they can be useful in fitting parameters used in other methods.

Another stream of literature [45 50] takes a multimodel perspective, integrating linear programming models that assume fixed, exogenous planned lead times with a detailed simulation model of the production system. An initial set of lead time estimates are used to derive an initial release plan from the LP model. The execution of this plan is then simulated, and realized values of the cycle times and other parameters of interest are collected. These observations are then used to update the planned lead times used in the LP model to obtain an updated release plan. The primary disadvantage of these techniques is the need 
for multiple replications of a detailed simulation model at each iteration of the planning procedure, which is likely to be prohibitive for large production systems. In addition, the convergence behavior of these methods is not yet well understood [50. Orcun et al. [51] implement a similar approach for production problems with stochastic demand.

The method proposed in this paper extends the clearing function model significantly, by providing a comprehensive statistical framework within which multiple time series representing the evolution of state variables over time can be used to predict the evolution of system output over time. While some efforts have been made to incorporate past history into the estimation of clearing functions [52], a rigorous statistical framework has hitherto been lacking, and only a very limited set of state variables have been considered. The proposed approach is based on data from extensive DES simulations, thus incorporating the detailed representation of stochastic interactions and constraints that DES provides; however, this knowledge is encoded in a metamodel that permits it to be used extremely efficiently in predicting the future evolution of the system.

\section{Methodology Overview}

In light of the limitations of the existing methods discussed above, we developed a metamodeling-based approach: The plan optimization is performed using a simulation optimization scheme in which the computationally expensive DES is replaced by the metamodelbased MCS to allow fast and accurate quantification of system input-output relationships. Metamodeling is used to bridge the gap between the detailed but highly time-consuming DES and the need for responsive decision making [53]. Once the configuration of a manufacturing system is established, its DES can be developed and kept running for weeks (or even months) to provide data for the estimation of the metamodels. The resulting metamodel not only embodies the high fidelity of DES, but also allows quick "what-if" analysis, and hence can be used to support responsive production optimization when the need for decision arises. The development of the metamodel-based MCS is the key contribution of this work, and serves as the foundation for responsive plan optimization.

Recall the input-output process illustrated in Figure 1. We define the following notation:

$\Delta t$ : the time interval considered as the basic time unit. All the time variables/parameters in this work are measured in terms of this time unit $\Delta t$.

$t$ : the time index measured in terms of the basic time unit.

$H$ : the length of planning horizon in terms of the basic time unit.

$A(t)$ : the input release (or arrival) process to the system which specifies the number of arrivals during the time interval $(t, t+1]$.

$x(t)=\mathrm{E}[A(t)]:$ the first moment of the arrival process $A(t)$. 
$Q(t)$ : the state process of the system which counts the number of units of work in process (WIP) in the system at time $t$.

$m_{i}(t)=\mathrm{E}\left[Q^{i}(t)\right]:$ the $i^{t h}$ moment of the state process $Q(t)(i=1,2)$.

$m_{1 j}(t)=\mathrm{E}[Q(t) Q(t-j)]$ : the first moment of $Q(t) Q(t-j)\left(j=1,2, \ldots, J_{Q}\right)$, where $J_{Q}$ is the highest order time lag needed to describe the WIP process.

$D(t)$ : the departure process from the system which counts the number of jobs finished during the time interval $(t, t+1]$.

$d_{i}(t)=\mathrm{E}\left[D^{i}(t)\right]$ : the $i^{t h}$ moment of the departure process $D(t)(i=1,2)$.

$d_{1 j}(t)=\mathrm{E}[D(t) D(t-j)]$ : the first moment of $D(t) D(t-j)\left(j=1,2, \ldots, J_{D}\right)$, where $J_{D}$ is the highest order time lag needed to describe the departures.

$e_{Q D}(t)=\mathrm{E}[Q(t) D(t)]:$ the first moment of $Q(t) D(t)$

$\mathfrak{D}(t)$ : the demand process which counts the number of items requested by customers at the end of period $t$.

The input $A(t)$ is assumed to be completely characterized by $x(t)$, which is a common assumption in production planning [54]. The output time series $Q(t)$ and $D(t)$ are assumed to be adequately characterized by their first two moments [55, 56]. The production planning task is thus to determine the release plan $\{x(t) ; t=1,2, \ldots, H\}$. The outputs, $\{Q(t), D(t) ; t=1,2, \ldots, H\}$, are bivariate time-series counts described by the characteristics vector

$$
\mathbf{y}(t)=\left(m_{1}(t), m_{2}(t), d_{1}(t), d_{2}(t), m_{11}(t), \ldots, m_{1 J_{Q}}(t), d_{11}(t), \ldots, d_{1 J_{D}}(t), e_{Q D}(t)\right)^{\top}
$$

The demand $\mathfrak{D}(t)$ is a given time-series pre-specified by forecasting methods outside of the scope of production planning. The output processes $\{Q(t), D(t) ; t=1,2, \ldots, H\}$, interact with the demand $\{\mathfrak{D}(t) ; t=1,2, \ldots, H\}$ to determine the system performance over $t=$ $1,2, \ldots, H$, which typically includes the total cost and fill rate.

We use a multi-objective genetic algorithm (MOGA) discussed in Section 6 to obtain near-optimal release plans. The performance metrics of a given candidate input schedule are evaluated using the two steps shown in Figure 2.

\begin{tabular}{|c|c|c|c|c|}
\hline \multirow{2}{*}{$\begin{array}{l}\text { Input } \\
\text { A release plan } \\
\text { of jobs }\end{array}$} & Metamodel & \multirow{2}{*}{$\begin{array}{c}\text { Outputs } \\
\text { Characteristics of the } \\
\text { system's output processes }\end{array}$} & Monte Carlo & \multirow{2}{*}{$\begin{array}{l}\text { Objective Metrics } \\
\text { (i): fill rate } \\
\text { (ii): mean and standard } \\
\text { deviation of cost }\end{array}$} \\
\hline & & & Simulation & \\
\hline
\end{tabular}

Figure 2: Evaluating a release plan's performance. 
Step (1): For an arbitrary release plan specified by $\left\{x^{*}(t) ; t=1,2, \ldots, H\right\}$, the pre-computed metamodel is used to predict the characteristics (1) of the output processes $\{Q(t), D(t) ; t=$ $1,2, \ldots, H\}$ over the planning horizon. As will be detailed in Section 4 , the metamodel takes the form of difference equations, is fitted from extensive DES data obtained offline (prior to performing plan optimization), and is able to accurately quantify the dependence of the output characteristics (1) upon the input release plan. The prediction can be performed extremely rapidly with the high-fidelity of DES, since the metamodel is a mathematical approximation derived from DES data.

Step (2): As pointed out in Section 1, the observed performance metrics result from the interactions of general time-series counts $\{Q(t), D(t) ; t=1,2, \ldots, H\}$ and $\{\mathfrak{D}(t) ; t=$ $1,2, \ldots, H\}$, and in general cannot be computed analytically. We develop an MCS method for time-series counts in Section 5 to simulate $\{Q(t), D(t) ; t=1,2, \ldots, H\}$ based on the output characteristics (1) obtained from Step (1), and to efficiently compute the performance metrics.

\section{Metamodeling via Offline Discrete-Event Simulation}

The metamodel in Step (1) of Figure 2 characterizes the time-dependent behavior of a general manufacturing system, and is obtained by extending the metamodeling method in [54]. In [54], the metamodel is fitted from DES data to quantify the relationship between $x(t)$, the first moment of the arrivals $A(t)$, and $\{m(t), d(t)\}$, the first-moment measures of the output processes $\{Q(t), D(t)\}$. As noted earlier, $\{Q(t), D(t)\}$ are bivariate timeseries counts, and thus their first-moment measures are not adequate to characterize these processes. In this paper, the metamodeling in [54] is extended to quantify the functional relationships between the input $x(t)$ and the $\mathbf{y}(t)$ vector, including both first- and secondmoment measures of $\{Q(t), D(t)\}$ as shown in (1). The metamodeling methods are described bellow, emphasizing the aspects different from those in [54].

\subsection{Functional Form of the Metamodel}

As in [54], the metamodel takes the form of difference equations, where the characteristics vector $\mathbf{y}(t)$ can be expressed as

$$
\mathbf{y}(t)=\mathbf{F}(x(t-1), x(t-2), \ldots, \mathbf{y}(t-1), \mathbf{y}(t-2), \ldots)
$$

where $\mathbf{F}$ is a vector function of compatible dimension with $\mathbf{y}(t)$. Each component function $F_{i}$ specifies the dependence of the $i^{t h}$ element of $\mathbf{y}(t)$ upon a given input $\{x(t-1), x(t-2), \ldots\}$ and the system's historical outputs $\{\mathbf{y}(t-1), \mathbf{y}(t-2), \ldots\}$. As in [54], $F_{i}$ assumes the functional form of a third-order polynomial. 


\subsection{Sampling via Discrete-Event Simulation (DES)}

To fit the metamodel (2) that functionally relates $x(t)$ to $\mathbf{y}(t)$, extensive DES experiments are carried out. For the DES, job arrivals are modeled by a time-varying process (e.g., nonstationary Poisson) characterized by its arrival rate $x(t)$. Each simulation run is performed by feeding the stochastic arrivals described by a pre-specified $x(t)$ into the system for a simulation period of $L$ time units $(\Delta t)$, and a total of $R$ simulation replications are obtained. The specifications of the input $x(t), L$ and $R$ are detailed in [54].

From the $r^{t h}$ replication, the arrival, WIP state and departure processes $\left\{A_{r}(t), Q_{r}(t)\right.$, $\left.D_{r}(t) ; t=1,2, \ldots, L\right\}$ are recorded. Based on the $R$ replications, the input-output data for the metamodel fitting are denoted by $\{(\widetilde{x}(t), \widetilde{\mathbf{y}}(t)) ; t=1,2, \ldots, L\}$ and calculated as follows:

$$
(\widetilde{x}(t), \widetilde{\mathbf{y}}(t))^{\top}=\left(\begin{array}{c}
\widetilde{x}(t) \\
\widetilde{m}_{1}(t) \\
\widetilde{m}_{2}(t) \\
\widetilde{d}_{1}(t) \\
\widetilde{d}_{2}(t) \\
\widetilde{m}_{11}(t) \\
\vdots \\
\widetilde{m}_{1 J_{Q}}(t) \\
\widetilde{d}_{11}(t) \\
\vdots \\
\widetilde{d}_{1 J_{D}}(t) \\
\widetilde{e}_{Q D}(t)
\end{array}\right)=R^{-1} \sum_{r=1}^{R}\left(\begin{array}{c}
A_{r}(t) \\
Q_{r}(t) \\
Q_{r}(t)^{2} \\
D_{r}(t) \\
D_{r}(t)^{2} \\
Q_{r}(t) Q_{r}(t-1) \\
\vdots \\
Q_{r}(t) Q_{r}\left(t-J_{Q}\right) \\
D_{r}(t) D_{r}(t-1) \\
\vdots \\
D_{r}(t) D_{r}\left(t-J_{D}\right) \\
Q_{r}(t) D_{r}(t)
\end{array}\right) .
$$

\subsection{Model Fitting and Selection}

From the data (3), the metamodel (2) is fitted using the method provided in Section 5.1 of [54]. The various model selection issues regarding the functional terms included in $\mathbf{F}$ of the metamodel are discussed in Section 5.2 of [54] . The additional model selection issue involved in modeling $\mathbf{y}(t)$, which includes not only the first-moment but also the second-moment measures of the output processes, lies in the determination of the time lags, $J_{Q}$ and $J_{D}$. Recall that $J_{Q}$ and $J_{D}$ are the highest orders of autocorrelation needed to characterize the processes $Q(t)$ and $D(t)$, respectively. Given the DES data $\left\{Q_{r}(t), D_{r}(t) ; t=1,2, \ldots, L ; r=\right.$ $1,2, \ldots, R\}, J_{Q}$ and $J_{D}$ can be determined by inspecting the sample partial autocorrelation function (SPACF) [57] of the time series.

\subsection{Metamodel-Based Prediction}

Once the metamodel has been obtained, it can be used to predict within a second the system's behavior under any input rate $x^{*}(t)$ over a time horizon. Specifically, suppose that we are currently standing at time 0 , where typically the system history $\{\mathbf{y}(t) ; t \leq 0\}$ 
is available. Using the historical outputs $\{\mathbf{y}(t) ; t \leq 0\}$ to initialize the metamodel-based computation, the future characteristic processes $\{\widehat{\mathbf{y}}(t) ; t=1,2, \ldots, H\}$ can be recursively predicted by the metamodel for any $\left\{x^{*}(t) ; t=1,2, \ldots, H\right\}$.

It is worth noting that the time to complete the metamodel-based recursive computation for future prediction is not sensitive to the complexity of the system being investigated, since the computation is performed based on the fitted metamodel, and thus involves only basic calculations such as additions and multiplications. This is because the metamodel is developed for the entire system, in contrast to the individual resources within the system as is the case for most clearing function models.

\section{Metamodel-Based Monte Carlo Simulation (MCS) for System Output Pro- cesses}

We now discuss Step (2) of Figure 2; For a release plan $\left\{x^{*}(t) ; t=1,2, \ldots, H\right\}$, how to evaluate the resulting system performance, having obtained the output characteristics $\{\widehat{\mathbf{y}}(t) ; t=1,2, \ldots, H\}$ from the metamodel prediction?

Since the performance metrics result from the interactions of the time series $\{Q(t), D(t) ; t=$ $1,2, \ldots, H\}$ and the customer demand $\{\mathfrak{D}(t) ; t=1,2, \ldots, H\}$ throughout the planing horizon, they cannot in general be evaluated analytically. Hence, an MCS method is developed in this work to link $\{\mathbf{y}(t) ; t=1,2, \ldots, H\}$ and the performance metrics. The MCS is able to quickly generate time-series $\{\widehat{Q}(t), \widehat{D}(t) ; t=1,2, \ldots, H\}$, whose major characteristics match the metamodel-predicted $\{\widehat{\mathbf{y}}(t) ; t=1,2, \ldots, H\}$. Clearly, the MCS-simulated processes $\{\widehat{Q}(t), \widehat{D}(t) ; t=1,2, \ldots, H\}$ are meant to mimic real (or DES) output processes $\{Q(t), D(t) ; t=1,2, \ldots, H\}$.

In this work, a parametric approach is taken to generate $\{\widehat{Q}(t), \widehat{D}(t) ; t=1,2, \ldots, H\}$ via MCS: First, a parametric model family of the output time series is identified (Section 5.1); Then, using the selected model family, the time-series model that possesses the metamodelpredicted characteristics $\{\widehat{\mathbf{y}}(t) ; t=1,2, \ldots, H\}$ is fitted (Section 5.2.1); Finally, based on the fitted time-series model, MCS is carried out to generate $\{\widehat{Q}(t), \widehat{D}(t) ; t=1,2, \ldots, H\}$ via pseudo-random generation mechanisms (Section 5.2.2).

\subsection{Time-Series Model Identification}

The model identification for the output time series is performed offline based on the DES data obtained for metamodeling. To establish the appropriate model for the bivariate process $(Q(t), D(t))^{\top}$, the first task is to identify suitable univariate time-series models that describe $Q(t)$ and $D(t)$. The selected univariate models are then combined to form a bivariate timeseries model for the joint processes $(Q(t), D(t))^{\top}$ by incorporating the correlation between $Q(t)$ and $D(t)$. 


\subsubsection{Candidate Models of Univariate Time Series}

For ease of exposition, define $Z(t)$ as a univariate time series representing either of the system output processes $Q(t)$ or $D(t)$. Our problem is to identify a family of univariate timeseries models suitable to describe $Z(t)$ ? First, $Z(t)$ is a counting process over time that takes non-negative integer values. Second, such a process from a general queueing system (e.g., manufacturing systems) could be over-dispersed, equi-dispersed or under-dispersed, i.e., the variance/mean ratio of $Z(t)$ could be greater than, equal to or less than 1 [58]. Third, the autocorrelations of $Z(t)$ could involve positive and/or negative values. Fourth, the selected model family should provide a straightforward relationship between its parameters and the process characteristics $\widehat{\mathbf{y}}(t)$, since the time-series model serves to generate MCS data $\{\widehat{Q}(t), \widehat{D}(t) ; t=1,2, \ldots, H\}$ that possess the given characteristics $\widehat{\mathbf{y}}(t)$.

In light of these requirements, we have identified three non-stationary time-series models as the potential candidates for $Z(t)$ : the N-AR, N-INAR and N-SINAR models [59 76]. Each of these models represents a non-stationary extension of its stationary counterpart by allowing the model parameters to vary with time. All can be written in the general autoregressive form:

$$
Z(t)=\sum_{j=1}^{J} \mathcal{R}\left(\alpha_{j}(t), Z(t-j)\right)+\varepsilon(t) ; \quad t=1,2, \ldots
$$

In (4), $J$ is a positive integer representing the order of the autoregressive model, $\varepsilon(t)$ denotes an independently-distributed innovation or shock, $\left\{\alpha_{j}(t) ; j=1,2, \ldots, J ; t=1,2, \ldots\right\}$ are time-dependent model parameters, and $\mathcal{R}$ a random operator. We assume that all the random operations in $\mathcal{R}$ are performed independently of each other and of the innovation process $\{\varepsilon(t) ; t=1,2, \ldots\}$.

The properties of these three models, which we now describe in order roof increasing complexity, are summarized in Table 1 .

Table 1: Summary of the candidate univariate time-series models.

\begin{tabular}{lcccc}
\hline & $\begin{array}{c}\text { Distribution } \\
\text { of } \varepsilon(t)\end{array}$ & $\begin{array}{c}\text { Feasible range } \\
\text { of } Z(t)\end{array}$ & $\begin{array}{c}\text { Able to accommodate negative } \\
\text { autocorrelations? }\end{array}$ & $\begin{array}{c}\text { Feasible range } \\
\text { of } \alpha_{j}(t)\end{array}$ \\
\hline N-AR & Normal & $\mathbb{R}$ & Yes & $(-$ inf, inf $)$ \\
N-INAR & GP & $\mathbb{N}$ & No & {$[0,1]$} \\
N-SINAR & GP & $\mathbb{Z}$ & Yes & {$[-1,1]$} \\
\hline
\end{tabular}

Model 1: The N-AR model extends the classic AR models [57] to incorporate time-varying parameters $\left\{\alpha_{j}(t) ; j=1,2, \ldots, J ; t=1,2, \ldots\right\}$ [77]. For N-AR, the general model (4) takes the specific form

$$
Z(t)=\sum_{j=1}^{J} \alpha_{j}(t) Z(t-j)+\varepsilon(t) ; \quad t=1,2, \ldots,
$$


where $Z(t) \in \mathbb{R}$, and $\varepsilon(t)$ is independently normally distributed.

Model 2: The N-INAR model extends the integer valued autoregressive (INAR) model [59, 61, 62] by incorporating time-varying parameters [78, 79]. The INAR model uses the binomial thinning operator and integer-valued innovations to generate integervalued time series. For N-INAR, the general model (4) takes the specific form

$$
Z(t)=\sum_{j=1}^{J} \alpha_{j}(t) \circ Z(t-j)+\varepsilon(t) ; \quad t=1,2, \ldots
$$

where $Z(t) \in \mathbb{N}$. The innovation $\varepsilon(t)$ is an independent non-negative integer-valued random variable, and controls the dispersion behavior of $Z(t)$ [61]. In order for N-INAR to model over-, equi- and under-dispersed data, the following discrete distributions could be used for $\varepsilon(t)$ : generalized Poisson (GP) [80], double Poisson [81, COMPoisson [82], Lerch distribution [61], or weighted Poisson [61]. In this work, we adopt the GP model for $\varepsilon(t)$ because this allows the distribution parameters of $\varepsilon(t)$ to be derived easily from the data characteristics $\widehat{\mathbf{y}}(t)$ and GP is widely used in modeling integer-valued time series [61, 75].

The operator "o" in (6) denotes the binomial thinning process [83] which is defined as

$$
\alpha \circ n=\sum_{m=1}^{n} C_{m} \text { for } n \geq 0
$$

where $\alpha \in[0,1]$ is the operation parameter and $C_{m}$ is a sequence of independent and identically distributed Bernoulli random variables independent of $Z(t)$, with $\mathbb{P}\left(C_{m}=\right.$ $1)=\alpha$. Since $\alpha$ takes the value of $\alpha_{j}(t)(j=1,2, \ldots, J)$ in $(6), \alpha_{j}(t)$ is restricted to be within the range of $[0,1]$; hence N-INAR only allows for time series with certain positive autocorrelation patterns [57].

Model 3: N-SINAR N-SINAR is the non-stationary extension of the signed integer-valued autoregressive (SINAR) model introduced in [67] and [84]. The general model (4) takes the form

$$
Z(t)=\sum_{j=1}^{J} F\left(\alpha_{j}(t)\right) \circ Z(t-i)+\varepsilon(t),
$$

where $Z(t) \in \mathbb{Z}$. The innovation $\varepsilon(t)$ is an independent integer-valued random variable. As with N-INAR, the GP distribution is used for $\varepsilon(t)$ in N-SINAR, and allows NSINAR to handle over-, equi- and under-dispersed data.

The operator " $F(\cdot) \circ$ " denotes the generalized thinning process 67

$$
F(\alpha) \circ n= \begin{cases}\operatorname{sign}(n) \sum_{m=1}^{|n|} C_{m} & \text { if } n \neq 0 \\ 0 & \text { otherwise }\end{cases}
$$


where $\alpha \in[-1,1]$ is the operation parameter; $\operatorname{sign}(n)$ equals to 1 if $n \geq 0$ and -1 if $n<0$; and $\left\{C_{m} ; m=1,2, \ldots\right\}$ are independent and identically distributed random variables with the probability distribution given as below [84]

$$
\mathbb{P}\left(C_{m}=-1\right)=\left(\frac{1-\alpha}{2}\right)^{2}, \mathbb{P}\left(C_{m}=0\right)=\frac{1-\alpha^{2}}{2}, \quad \mathbb{P}\left(C_{m}=1\right)=\left(\frac{1+\alpha}{2}\right)^{2}
$$

Since $\alpha$ takes the value of $\alpha_{j}(t)(j=1,2, \ldots, J)$ in $(8), \alpha_{j}(t)$ is restricted to the range of $[-1,1]$; thus, N-SINAR can model integer-valued time series with both positive and negative serial correlations due to its flexible thinning operators (9).

\subsubsection{Model Selection for Univariate Time Series}

From extensive offline DES (Section 4.2), the time-series data for an output process can be obtained as $\left\{Z_{r}(t) ; t=1,2, \ldots, L ; r=1,2, \ldots, R\right\}$. Recall that $L$ denotes the simulation length of each replication, and $R$ the number of replications. $Z_{r}(t)$ could be the WIP state $Q_{r}(t)$ or the departure $D_{r}(t)$ as denoted in Section 4.2 . As discussed in 4.3 . the autoregressive order $J$ in model (4) can be determined based on the data $\left\{Z_{r}(t) ; t=\right.$ $1,2, \ldots, L ; r=1,2, \ldots, R\}$.

The question remaining to be answered is: How to select the most appropriate of the three models (N-AR, N-INAR and N-SINAR) to describe the data? Both transient and steady-state data are included in $\left\{Z_{r}(t) ; t=1,2, \ldots, L ; r=1,2, \ldots, R\right\}$ following the design of DES experiments in [54]. For convenience, only the steady-state data are used for timeseries model selection. In the spirit of finding the simplest and adequate model, we provide the following guidelines.

First, the steady-state data are subjected to normality tests, such as QQ-plot [85], Kolmogorov-Smirnov test [86] or Shapiro-Wilk test [87], to determine whether N-AR is appropriate. Although the time series are non-negative integer counts, the simplest N-AR may provide an adequate fit, especially when the counts are large. If the data fail the normality tests, the integer-valued models (N-INAR and N-SINAR) are considered.

Due to its relative simplicity, N-INAR is favored over N-SINAR unless it is inadequate to capture important data features. As shown in Table 1, N-INAR and N-SINAR differ in terms of the feasible range of the parameters $\left\{\alpha_{j}(t) ; j=1,2, \ldots, J\right\}$, which allows them to model different autocorrelation patterns. Hence, the estimation and inference of $\left\{\alpha_{j} ; j=1,2, \ldots, J\right\}$ are used to guide the model selection between INAR and SINAR. More specifically, the sampling autocorrelation function (SACF) can be calculated from the stationary DES data and fed to the Yule-Walker equation [57, 88] to obtain estimates of $\left\{\alpha_{j} ; j=1,2, \ldots, J\right\}$. Inferences on the Yule-Walker estimates can also be derived, and the following hypothesis test performed:

$$
H_{0}: \alpha_{j} \geq 0 ; \quad \text { vs. } H_{a}: \alpha_{j}<0
$$


for $j=1,2, \ldots, J$. If $H_{0}$ is rejected for any $j$, N-SINAR is selected; otherwise, N-INAR is adopted. The details for the Yule-Walker estimation and inference are given in Appendix C.

\subsubsection{Bivariate Time-Series Model}

After appropriate univariate time-series models are selected for each of the two processes, $Q(t)$ and $D(t)$, these can then be used to form the bivariate time-series model for $(Q(t), D(t))^{\top}$.

The bivariate time series $(Q(t), D(t))^{\top}$ is modeled as:

$$
\begin{aligned}
& Q(t)=\sum_{j=1}^{J_{Q}} \mathcal{R}^{(Q)}\left(\alpha_{j}^{(Q)}(t), Q(t-j)\right)+\varepsilon^{(Q)}(t) \\
& D(t)=\sum_{j=1}^{J_{D}} \mathcal{R}^{(D)}\left(\alpha_{j}^{(D)}(t), D(t-j)\right)+\varepsilon^{(D)}(t)
\end{aligned}
$$

where $\boldsymbol{\varepsilon}(t)=\left(\varepsilon^{(Q)}(t), \varepsilon^{(D)}(t)\right)^{\top}$ is the innovation vector with

$$
\operatorname{Var}[\varepsilon(t)]=\left(\begin{array}{cc}
\operatorname{Var}\left[\varepsilon^{(Q)}(t)\right] & \operatorname{Cov}\left[\varepsilon^{(Q)}(t), \varepsilon^{(D)}(t)\right] \\
\operatorname{Cov}\left[\varepsilon^{(Q)}(t), \varepsilon^{(D)}(t)\right] & \operatorname{Var}\left[\varepsilon^{(D)}(t)\right]
\end{array}\right)
$$

In the bivariate model, the marginal distributions of $\varepsilon^{(Q)}(t)$ and $\varepsilon^{(D)}(t)$ are specified by the corresponding univariate time-series model (Section 5.1.1); $J_{Q}$ and $J_{D}$ are the orders of the univariate autoregressive models; and the specific forms of the random operators, $\mathcal{R}^{(Q)}$ and $\mathcal{R}^{(D)}$, correspond to the univariate models selected for $Q(t)$ and $D(t)$, respectively.

\subsection{Metamodel-Based Monte Carlo Simulation}

As can be seen from Section 5.1, a bivariate time-series model family $(11,12$ can be selected for the target processes $(Q(t), D(t))^{\top}$ based on offline DES. The selected model can then be fitted from the given characteristics $\widehat{\mathbf{y}}(t)$ (Section 5.2.1). The fitted bivariate model allows for the MCS of $(\widehat{Q}(t), \widehat{D}(t))^{\top}$ (Section 5.2.2.

\subsubsection{Time-Series Model Fitting}

Denote the fitted bivariate time-series model as

$$
\begin{aligned}
& \widehat{Q}(t)=\sum_{j=1}^{J_{Q}} \mathcal{R}^{(Q)}\left(\widehat{\alpha}_{j}^{(Q)}(t), \widehat{Q}(t-j)\right)+\widehat{\varepsilon}^{(Q)}(t) \\
& \widehat{D}(t)=\sum_{j=1}^{J_{D}} \mathcal{R}^{(D)}\left(\widehat{\alpha}_{j}^{(D)}(t), \widehat{D}(t-j)\right)+\widehat{\varepsilon}^{(D)}(t) .
\end{aligned}
$$

The fitted parameters include $\left\{\widehat{\alpha}_{j}^{(Q)}(t) ; j=1,2, \ldots, J_{Q}\right\},\left\{\widehat{\alpha}_{j}^{(D)}(t) ; j=1,2, \ldots, J_{D}\right\}, \widehat{\mathrm{E}}\left[\widehat{\varepsilon}^{(Q)}(t)\right]$, $\widehat{\mathrm{E}}\left[\widehat{\varepsilon}^{(D)}(t)\right], \widehat{\operatorname{Var}}\left[\widehat{\varepsilon}^{(Q)}(t)\right], \widehat{\operatorname{Var}}\left[\widehat{\varepsilon}^{(D)}(t)\right]$ and $\widehat{\operatorname{Cov}}\left[\widehat{\varepsilon}^{(Q)}(t), \widehat{\varepsilon}^{(D)}(t)\right]$, which can be derived analyti- 
cally as functions of the metamodel-predicted characteristics $\widehat{\mathbf{y}}(t)$ as shown in Appendix D.

Hence, the bivariate process described by the fitted model possesses the same characteristics as those specified by $\widehat{\mathbf{y}}(t)$. Specifically,

- The marginal mean, variance, and lag- $j\left(j=1,2, \ldots, J_{Q}\right)$ autocorrelations of $\widehat{Q}(t)$ are the same as those specified by $\widehat{\mathbf{y}}(t)$; the same can be concluded for $\widehat{D}(t)$.

- The covariance of $\widehat{Q}(t)$ and $\widehat{D}(t)$ is the same as that specified by $\widehat{\mathbf{y}}(t)$.

\subsubsection{Monte Carlo Simulation of the System Output Processes}

The fitted bivariate model $14-15)$ can be used to perform MCS to generate $(\widehat{Q}(t), \widehat{D}(t))^{\top}$ time series mimicking the real WIP-state and departure processes.

The historical data $\left\{Q(t), D(t) ; t=0,-1, \ldots,-\max \left(J_{Q}, J_{D}\right)+1\right\}$ is typically available to initialize the computations $140-(15)$. With given mean vector $\left(\widehat{\mathrm{E}}\left[\widehat{\varepsilon}^{(Q)}(t)\right], \widehat{\mathrm{E}}\left[\widehat{\varepsilon}^{(D)}(t)\right]\right)^{\top}$ and variance-covariance matrix

$$
\widehat{\operatorname{Var}}[\varepsilon(t)]=\left(\begin{array}{cc}
\widehat{\operatorname{Var}}\left[\widehat{\varepsilon}^{(Q)}(t)\right] & \widehat{\operatorname{Cov}}\left[\widehat{\varepsilon}^{(Q)}(t), \widehat{\varepsilon}^{(D)}(t)\right] \\
\widehat{\operatorname{Cov}}\left[\widehat{\varepsilon}^{(Q)}(t), \widehat{\varepsilon}^{(D)}(t)\right] & \widehat{\operatorname{Var}}\left[\widehat{\varepsilon}^{(D)}(t)\right]
\end{array}\right),
$$

the bivariate innovation process $\left(\widehat{\varepsilon}^{(Q)}(t), \widehat{\varepsilon}^{(D)}(t)\right)^{\top}$ can be simulated. As explained in Section 5.1.1, we assume a univariate innovation follows a continuous normal or discrete GP distribution. If both $\widehat{\varepsilon}^{(Q)}(t)$ and $\widehat{\varepsilon}^{(D)}(t)$ are normally distributed, the bivariate innovations can be easily generated by the simulation algorithms for multivariate normal distributions [89]. If both innovation processes follow discrete GP, the copula-based algorithm in [90] can be used for simulation. Otherwise, if one is discrete and the other is continuous, the algorithm in [91] can be employed. The copula-based algorithms developed in [90, 91] are given in Appendix E for the readers' convenience.

\section{Metamodel-Based Optimization for Online Production Planning}

Optimization of production planning is performed using the capability of the metamodelbased MCS to very rapidly and accurately evaluate the performance measures for a given input release plan.

\subsection{Problem Formulation of Production Planning}

The plan optimization problem is formulated following the basic structure in the existing production literature [14, 33, 36, 37, 41, 52]. The problem formulation also represents an extension for generality, which is allowed by our metamodel-based methods.

Following the notation in Section 3 , the planning horizon is denoted as $(0, H]$ and divided into $P$ equal-length time periods. $H$ is given in terms of the number of time units $\Delta t$. The length of each period is set based on the problem environment. Within each time period, 
the job release rate is assumed to be constant and equal to $x_{p}$ with $p=1,2, \ldots, P$. Thus, the release plan over $(0, H]$ can be specified by a $P \times 1$ vector

$$
\mathbf{x}=\left(x_{1}, x_{2}, \ldots, x_{P}\right)^{\top}
$$

which constitutes the vector of decision variables for plan optimization. The customer demand is a given stochastic time series provided by forecasting efforts outside of the scope of production planning. As in Section 3 , the demand is denoted by $\{\mathfrak{D}(t) ; t=1,2, \ldots, H\}$. Depending on when demand is actually fulfilled (e.g., if shipments are made only at the end or beginning of a period), $\mathfrak{D}(t)$ could be set to 0 at certain time points.

The parameters, dependent variables, and objective functions of the optimization problem are provided below.

\section{Parameters}

$w_{t}$ the unit holding cost of the WIP per time unit at time $t$.

$h_{t}$ the unit holding cost of finished jobs (or products) per time unit at time $t$.

$G_{0}$ the initial number of finished jobs at time 0 , the beginning of the planning horizon.

\section{Dependent Variables}

- The output processes from the system, defined in Section 3 and rewritten here to stress their dependence on $\mathbf{x}$ :

$$
\{Q(t, \mathbf{x}), D(t, \mathbf{x}) ; t=1,2, \ldots, H\}
$$

Accordingly, the characteristics of (17) are denoted by:

$$
\begin{aligned}
\mathbf{y}(t, \mathbf{x})= & \left(m_{1}(t, \mathbf{x}), m_{2}(t, \mathbf{x}), d_{1}(t, \mathbf{x}), d_{2}(t, \mathbf{x}), m_{11}(t, \mathbf{x}), \ldots, m_{1 J_{Q}}(t, \mathbf{x})\right. \\
& \left.d_{11}(t, \mathbf{x}), \ldots, d_{1 J_{D}}(t, \mathbf{x}), e_{Q D}(t, \mathbf{x})\right)^{\top}
\end{aligned}
$$

whose $\mathbf{x}$-free correspondence is defined in Section 3 .

- $G(t, \mathbf{x})$ : The number of finished jobs at the end of the $t^{t h}$ time unit, after the inventory has been refilled by the newly-completed jobs and demand (if any) has been met as far as possible in that time unit. It is calculated as

$$
G(t, \mathbf{x})=G(t-1, \mathbf{x})+D(t, \mathbf{x})-\mathfrak{D}(t) ; \quad t=1,2, \ldots, H
$$

and is a time series resulting from the interaction of the two stochastic time series $\{D(t, \mathbf{x}) ; t=1,2, \ldots, H\}$ and $\{\mathfrak{D}(t) ; t=1,2, \ldots, H\}$. In this formulation, $G(t, \mathbf{x})$ is allowed to be a negative integer, representing the backlogged shortage by the end of the $t^{\text {th }}$ time unit. 
- $I(t, \mathbf{x})$ : The on-hand inventory of finished jobs at the end of the $t^{\text {th }}$ time unit, which is given as:

$$
I(t, \mathbf{x})=\max \{G(t, \mathbf{x}), 0\} ; \quad t=1,2, \ldots, H .
$$

- $B(t, \mathbf{x})$ : The number of customer demands that are realized in the $t^{\text {th }}$ time unit but cannot be satisfied immediately with the existing inventory. These demands will be backlogged and fulfilled on a first-come-first-serve basis.

$$
B(t, \mathbf{x})= \begin{cases}\mathfrak{D}(t) & \text { if } G(t-1, \mathbf{x})+D(t, \mathbf{x})<0 \\ -\min \{G(t-1, \mathbf{x})+D(t, \mathbf{x})-\mathfrak{D}(t), 0\} & \text { otherwise }\end{cases}
$$

where $t=1,2, \ldots, H$.

\section{Objective Criteria}

The total cost associated with a release plan $\mathbf{x}$ can be written as:

$$
T C(\mathbf{x})=\sum_{t=1}^{H} w_{t} Q(t, \mathbf{x})+\sum_{t=1}^{H} h_{t} I(t, \mathbf{x}) ; t=1,2, \ldots, H
$$

including the WIP holding cost and the finished-goods inventory cost. The total cost $T C(\mathbf{x})$ is a random variable depending on the release plan $\mathbf{x}$, and it is of interest to quantify both its expectation $\mathrm{E}[T C(\mathbf{x})]$ and its standard deviation $\operatorname{Std}[T C(\mathbf{x})]$.

The backlogged shortage $\{B(t, \mathbf{x}) ; t=1,2, \ldots, H\}$ can be used to obtain the fill rate $F R(\mathbf{x})$, the percentage of customer demand that can be fulfilled immediately with existing inventory, using the expression:

$$
F R(\mathbf{x})=\frac{\sum_{t=1}^{H} B(t, \mathbf{x})}{\sum_{t=1}^{H} \mathfrak{D}(t)} ; t=1,2, \ldots, H .
$$

The objectives of production planning could be to minimize $\mathrm{E}[T C(\mathbf{x})]$ and/or to maximize $\mathrm{E}[F R(\mathbf{x})]$.

This formulation represents only one possible way to formulate the optimization problem of production planning [1]. Our metamodel-based MCS method can accommodate a wide range of formulations, depending on the practical needs.

\subsection{Solving the Optimization Problem}

Figure 3 outlines the procedure to search for the production plans that combine good cost and service performance. This online production planning is performed with the two givens obtained offline: (a) the metamodel relating a release plan $\mathbf{x}\{\widehat{\mathbf{y}}(t, \mathbf{x}) ; t=1,2, \ldots, H\}$, the characteristics of the output processes $\{Q(t), D(t) ; t=1,2, \ldots, H\}$; (b) the bivariate time-series model selected based on the offline DES data. 
To consider multiple objective criteria, a multi-objective optimization algorithm such as gamultiobj in Matlab Optimization Toolbox is used to search the decision space of $\mathbf{x}$ for Pareto optimal solutions (release plans) as shown in Step (v) of Figure 3.

Each candidate plan $\mathbf{x}$ is evaluated rapidly and accurately through Steps (i)-(iv). For a given $\mathbf{x}$, Step (i) can typically be completed within a second to obtain $\{\widehat{\mathbf{y}}(t, \mathbf{x}) ; t=$ $1,2, \ldots, H\}$ over the planning horizon. Using $\{\widehat{\mathbf{y}}(t, \mathbf{x}) ; t=1,2, \ldots, H\}$, Step (ii) is to obtain the fitted bivariate time-series model for $(Q(t), D(t))^{\top}$.

In Step (iii), MCS is performed: Based on the fitted time-series model, $\left\{\widehat{Q}_{r}(t), \widehat{D}_{r}(t) ; t=\right.$ $1,2, \ldots, H ; r=1,2, \ldots, R\}$ is generated, with $R$ denoting the number of simulation replications; and by using the demand forecasting model, the demand series $\left\{\widehat{\mathfrak{D}}_{r}(t) ; r=1,2, \ldots, R\right\}$ is also simulated. The number of replications $R$ required for each $\mathbf{x}$ to obtain estimates of $\mathrm{E}[T C(\mathbf{x})], \operatorname{Std}[T C(\mathbf{x})]$, and $\mathrm{E}[F R(\mathbf{x})]$ with desired statistical precision is determined by a two-stage procedure [41, 54] given in Appendix F.

In Step (iv), the performance metrics $\widehat{\mathrm{E}}[T C(\mathbf{x})], \widehat{\operatorname{Std}}[T C(\mathbf{x})]$ and $\widehat{\mathrm{E}}[F R(\mathbf{x})]$ are obtained from the multiple replications of MCS data $\left\{\widehat{Q}_{r}(t), \widehat{D}_{r}(t) ; \widehat{\mathfrak{D}}_{r}(t) ; t=1,2, \ldots, H ; r=\right.$ $1,2, \ldots, R\}$.

On a computer with Intel(R) Core(TM) i7 CPU and 8G RAM, it takes 0.1 second on average to perform one MCS replication, while one DES replication for the sample system in Section 7 takes about 1 second. It is worth pointing out that the DES time depends on the complexity of the real system of interest, whereas the MCS time per replication remains approximately unchanged regardless of the system complexity. 


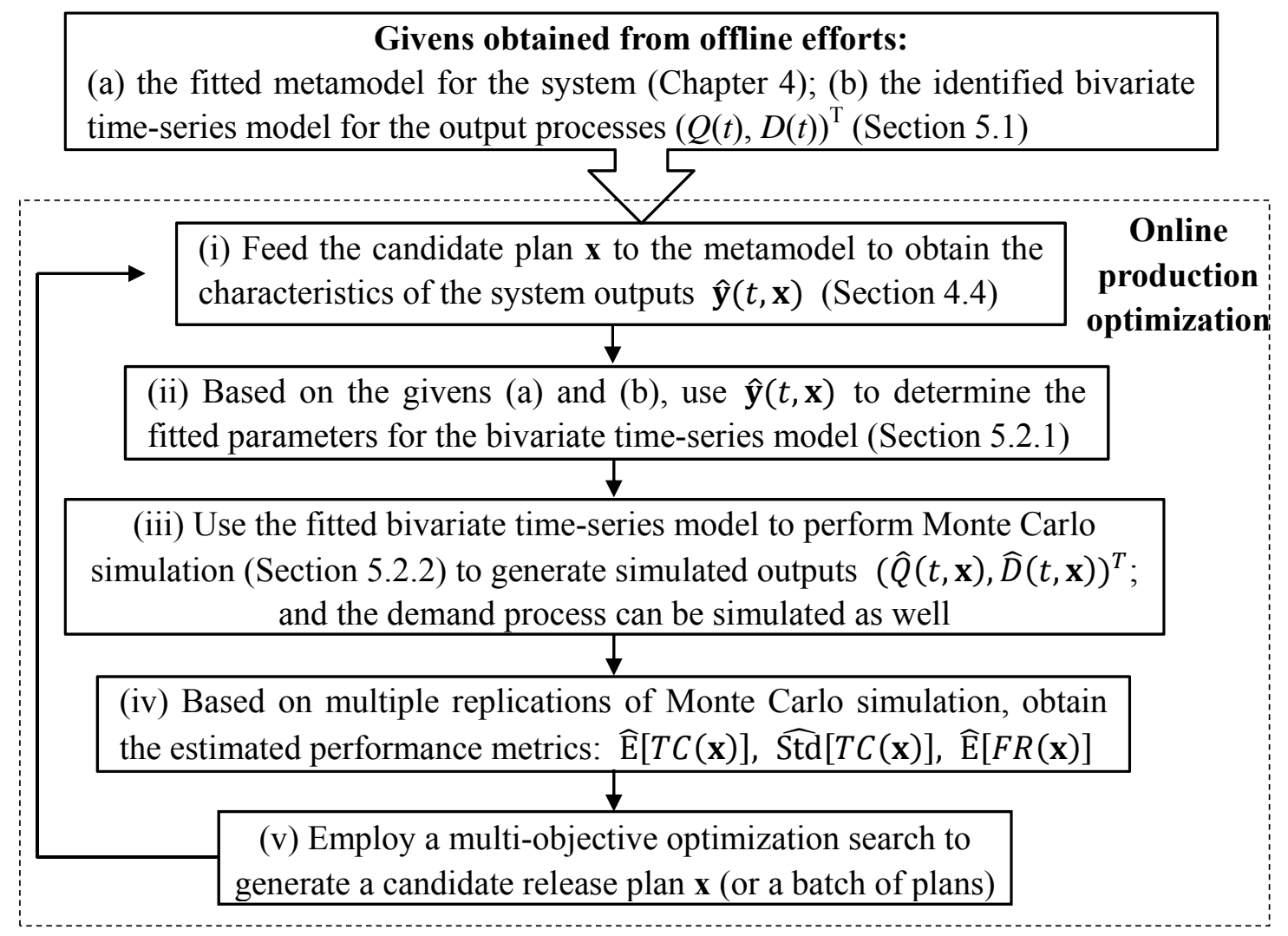

Figure 3: Online optimization for production planning.

\section{Empirical Results}

For purposes of demonstration, the proposed method has been applied to solve the production planning problem for a scaled-down semiconductor wafer fabrication system 92 described in Appendix A. The system consists of 9 workstations, and involves reentrant flows, machine failures and batch processing, which are the main features of a real semiconductor fabrication system. One type of job is considered and the job arrivals follow a Poisson distribution with time-varying arrival rates specified by a release plan. Each job has 14 processing steps and needs to visit some workstations more than once. The raw processing time (excluding waiting time) for a job is expected to be 12.9 hours, and the basic time unit $\Delta t=2$ hours.

The production planning problem is specified as follows for this case. The length of the planning horizon $H$ is set as 672 time units (eight weeks) and divided into eight equallength time periods, with each one 84 time units (one week) long. The cost parameters are assumed to be time-independent with values: $w=1$ and $h=2$, following [54, 93]. The initial number of finished jobs $G_{0}=0$. The customer demand $\{\mathfrak{D}(t) ; t=1,2, \ldots, H\}$ is a forecasted time series, which in this case is modeled as an AR-GARCH process, a widelyused time-series model for demand forecasting [94 97]. The simulation algorithm for an AR-GARCH is given in Appendix G. In this study, four AR-GARCH demand processes are considered, with expected values and realization times of demand following the four 


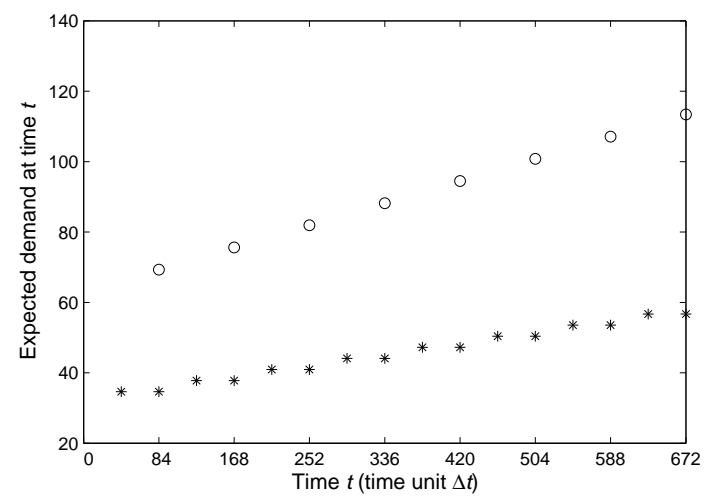

(a) Scenarios 1 and 2

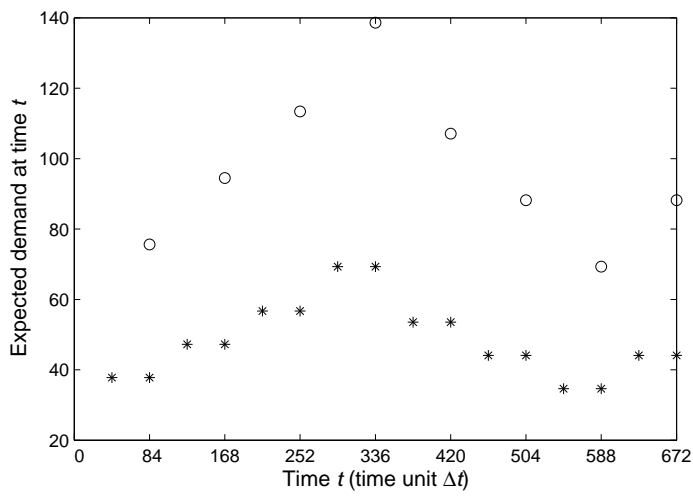

(b) Scenarios 3 and 4

Figure 4: Expected demand over the planning horizon of 672 time units (i.e., 8 weeks): "o" corresponds to Scenario 1 or 3 ; “*” corresponds to Scenario 2 or 4 .

scenarios in Figure 4. In Scenario 1, the expected weekly demand increases steadily over the planning horizon, and customer demand is realized once a week; Scenario 2 also follows the general increasing pattern, but allows the demand to be realized twice a week. Scenarios 3 and 4 also correspond to different frequencies of demand realization (as Scenarios 1 and 2 do); their expected demands fluctuate over the time. The values of expected weekly demand for each scenario are given later in Table 3 . In Scenarios 3 and 4, the average demand rate in the $4^{\text {th }}$ week exceeds the system capacity.

\subsection{Offline Simulation and Modeling Efforts}

Based on the DES data obtained offline, the metamodel was fitted (Section 4), with the autoregressive order $J_{Q}$ and $J_{D}$ both determined as 2 . In Appendix B, the fitted metamodel for this sample system is provided and evaluated. Following Section 5.1, N-AR and N-INAR were identified as appropriate univariate time-series models for $Q(t, \mathbf{x})$ and $D(t, \mathbf{x})$ respectively. The fitted metamodel and identified time-series models serve as the basis for online production optimization, as shown in Figure 3.

\subsection{Evaluation of Release Plans}

For online production optimization, a candidate plan needs to be evaluated using the metamodel-based MCS approach per Steps (i)-(iv) of Figure 3. To evaluate the accuracy of the MCS evaluation, an extremely large number of DES replications were performed for each release plan to evaluate the performance metrics with very high precision; the DES estimates can thus be considered as "true" values for the performance metrics.

Table 2 compares the estimates of expected total cost $\mathrm{E}[T C(\mathbf{x})]$, standard deviation of total cost $\operatorname{Std}[T C(\mathbf{x})]$ and expected fill rate $\mathrm{E}[F R(\mathbf{x})]$, obtained via the MCS method and DES respectively, for several different release plans. A total of 12 release plans are considered, derived from the four demand scenarios (Figure 4). For instance, the first plan 
in Table 2 is denoted as "Scenario $1 \times 1.05$ ", which means that the expected number of jobs weekly released under this plan is 1.05 times the expected weekly demand of Scenario 1. The table indicates that the MCS method is able to provide highly accurate estimates for the performance metrics of interest.

Table 2: Evaluation quality of the metamodel-based Monte Carlo Simulation (MCS).

\begin{tabular}{|c|c|c|c|c|c|c|c|c|c|}
\hline & \multicolumn{3}{|c|}{$\widehat{\mathrm{E}}[T C(\mathbf{x})]$} & \multicolumn{3}{|c|}{$\widehat{\operatorname{Std}}[T C(\mathbf{x})]$} & \multicolumn{3}{|c|}{$\widehat{\mathrm{E}}[F R(\mathbf{x})]$} \\
\hline Release plan $\mathbf{x}$ & MCS & DES & Deviation & MCS & DES & Deviation & MCS & DES & Deviation \\
\hline Scenario $1 \times 1.05$ & 95,369 & 95,037 & $0.35 \%$ & 35,597 & 33,211 & $7.18 \%$ & $92.77 \%$ & $93.59 \%$ & $-0.88 \%$ \\
\hline Scenario $1 \times 1$ & 74,153 & 74,666 & $-0.69 \%$ & 31,667 & 30,522 & $3.75 \%$ & $85.30 \%$ & $86.70 \%$ & $-1.61 \%$ \\
\hline Scenario $1 \times 0.95$ & 60,643 & 59,546 & $1.84 \%$ & 27,895 & 26,182 & $6.54 \%$ & $77.55 \%$ & $77.26 \%$ & $0.38 \%$ \\
\hline Scenario $2 \times 1.05$ & 65,306 & 66,453 & $-1.73 \%$ & 36,318 & 35,188 & $3.21 \%$ & $85.11 \%$ & $86.86 \%$ & $-2.01 \%$ \\
\hline Scenario $2 \times 1$ & 48,940 & 47,960 & $2.04 \%$ & 31,963 & 30,565 & $4.57 \%$ & $74.49 \%$ & $75.04 \%$ & $-0.73 \%$ \\
\hline Scenario $2 \times 0.95$ & 37,458 & 37,699 & $-0.64 \%$ & 26,123 & 25,977 & $0.56 \%$ & $64.59 \%$ & $65.31 \%$ & $-1.10 \%$ \\
\hline Scenario $3 \times 1.05$ & 84,354 & 83,820 & $0.64 \%$ & 33,202 & 31,448 & $5.58 \%$ & $87.18 \%$ & $87.94 \%$ & $-0.86 \%$ \\
\hline Scenario $3 \times 1$ & 65,319 & 65,962 & $-0.97 \%$ & 28,937 & 28,423 & $1.81 \%$ & $77.65 \%$ & $78.55 \%$ & $-1.15 \%$ \\
\hline Scenario $3 \times 0.95$ & 52,677 & 52,654 & $0.04 \%$ & 23,989 & 23,284 & $3.03 \%$ & $68.77 \%$ & $69.10 \%$ & $-0.48 \%$ \\
\hline Scenario $4 \times 1.05$ & 55,488 & 55,512 & $-0.04 \%$ & 32,933 & 33,523 & $-1.76 \%$ & $77.69 \%$ & $77.90 \%$ & $-0.27 \%$ \\
\hline Scenario $4 \times 1$ & 41,550 & 42,347 & $-1.88 \%$ & 28,066 & 28,754 & $-2.39 \%$ & $66.24 \%$ & $66.71 \%$ & $-0.70 \%$ \\
\hline Scenario $4 \times 0.95$ & 31,093 & 30,569 & $1.71 \%$ & 21,804 & 21,840 & $-0.16 \%$ & $54.91 \%$ & $54.01 \%$ & $1.67 \%$ \\
\hline
\end{tabular}

\subsection{Optimization Results}

We consider the production planning as a two-objective optimization problem which seeks to minimize the expected total cost $\mathrm{E}[T C(\mathbf{x})]$ and maximize the expected fill rate $\mathrm{E}[F R(\mathbf{x})]$ simultaneously. As a multi-objective optimization problem with conflicting objectives, we seek a set of non-dominated solutions that are as close as possible to the Pareto-optimal front and that are as diverse as possible [98] to allow decision makers to weigh the trade-offs.

The MOGA function "gamultiobj" provided by Matlab is adopted to perform a search in the decision space of $\mathbf{x}$. This function employs the Elitist Non-Dominated Sorting GA (NSGA-II) algorithm, which is widely used for multi-objective optimization problem [98]. The user-specified parameters for the algorithm include: population size, maximum number of generation, stopping criteria, mutation function, elite count, and initial population. In our work, the initial population is generated by combining a fractional factorial design and the default space-filling design in "gamultiobj" to provide a good coverage of the $\mathbf{x}$ space. The population size and maximum number of generations are set to 75 and 8, respectively, and the other parameters are left as their default values in Matlab.

The production plan optimization was performed for the four demand scenarios (shown in Figure 4) respectively. It takes about 50 hours to complete the MCS-based optimization for a given demand scenario on a computer with Intel(R) Core(TM) i7 CPU and 8G RAM. In comparison, the same optimization procedure takes about 500 hours, if the MCS evaluation is replaced by the DES for this scale-down fabrication system; the time savings will be more pronounced for systems with higher complexity, since DES time is very sensitive to system complexity whereas MCS is not.

For each demand scenario, a number of non-dominated solutions were obtained, and each solution's performance pair $(\widehat{E}[T C(\mathbf{x})], \widehat{E}[F R(\mathbf{x})])$ plotted in Figure 5 . Appendix H 


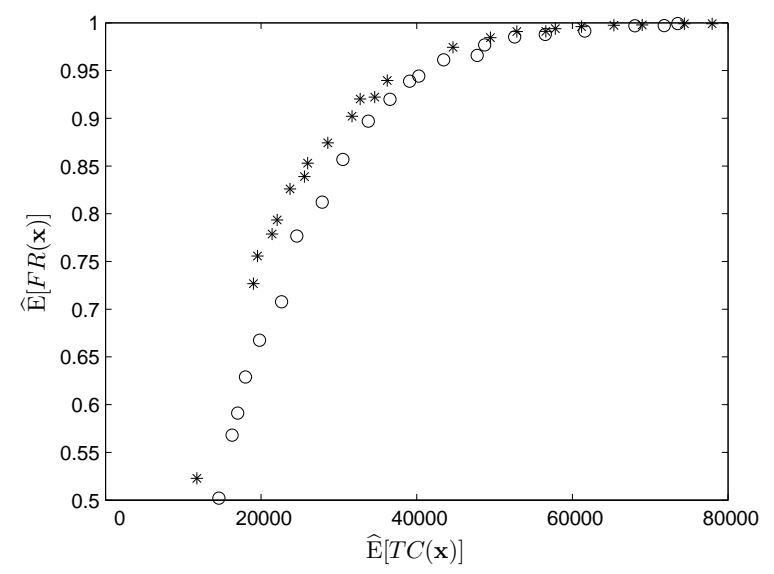

(a) Scenarios 1 and 2

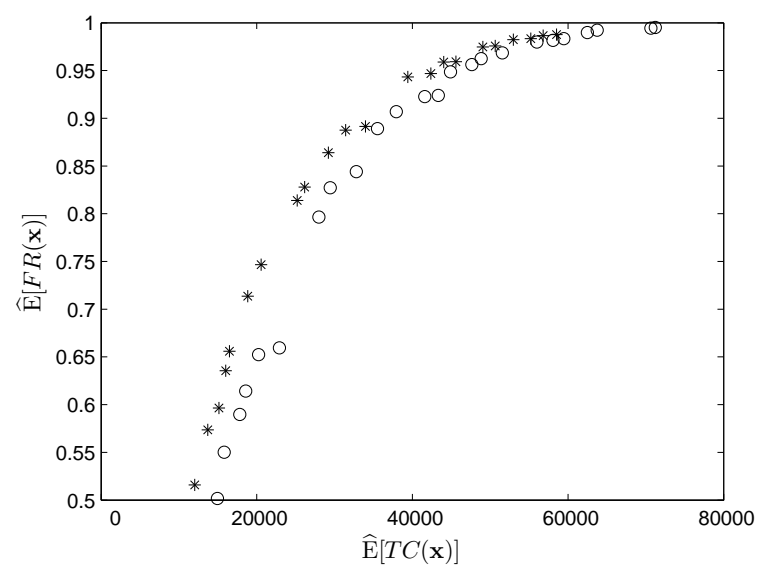

(b) Scenarios 3 and 4

Figure 5: Performance metrics of the non-dominated solutions obtained from the multi-objective optimization for each demand scenario: "o" corresponds to Scenario 1 or 3 ; "*" corresponds to Scenario 2 or 4.

briefly discusses the behavior of the NSGA-II algorithm based on our empirical results.) The four Pareto fronts of non-dominated solutions provide similar coverage of the performance region: The expected total cost ranges from 12, 879 to 194, 925, and the fill rate from $18.82 \%$ to $99.91 \%$, providing decision makers extensive information on the trade-offs between cost and customer satisfaction.

As shown in Figure 5 (a) or (b), the Pareto front depicted by the stars (for Scenarios 2 or 4 ) is slightly higher than that represented by the circles (for Scenarios 1 or 3): With the same cost, a higher fill rate can be achieved for Scenario 2 (or 4), compared to Scenario 1 (or 3). This is because the plan optimization is able to match the job outputs with the more frequently-realized (biweekly) customer demand in Scenario 2 (or 4), leading to lower finished-goods inventory than the cases (Scenario 1 or 3) where demand is realized weekly.

Table 3 provides two non-dominated solutions (represented by eight-element row vectors in the table) for each demand scenario, with fill rates approximately $95 \%$ and $99 \%$, the high levels that are usually of interest. For comparison purposes, the release plan that keeps the system running at $95 \%$ utilization over the planning horizon is also given in Table 3 , with the expected number of jobs released each week being $(120,120,120,120,120,120,120,120)$ during the period. This release plan leads to very high fill rate and cost, as expected. The selected solutions (the row vectors in Table 3 representing the expected number of jobs each week over the horizon) are compared with the expected weekly demand under their corresponding demand scenarios. It appears that to achieve a fill rate of about $95 \%$, these solutions tend to follow the general trend of customer demand (increasing for Scenario 1 and 2, and fluctuating for Scenario 3 and 4), but almost always over-produce a little in each week. This pattern becomes less obvious in the solutions leading to a fill rate of about $99 \%$, which is achieved by more pronounced over-production at substantially higher total cost. Given the high level of utilization required, this is not surprising; there is very little excess 
capacity to allow the system to respond rapidly to high demand, requiring inventories to be built up in earlier periods with lower demand, and the total demand over the horizon requires a high level of production in all periods.

Table 3: Selected non-dominated solutions obtained from multi-objective optimization for each demand scenario.

\begin{tabular}{|c|c|c|c|c|c|c|c|c|c|c|}
\hline & \multicolumn{10}{|c|}{ Expected weekly demand of Scenarios 1 and 2} \\
\hline & 69 & 76 & 82 & 88 & 95 & 101 & 107 & 113 & & \\
\hline & \multicolumn{8}{|c|}{ Expected number of jobs released each week } & $\widehat{E}[T C(\mathbf{x})]$ & $\widehat{\mathrm{E}}[F R(\mathbf{x})]$ \\
\hline \multirow{3}{*}{ Scenario 1} & 76 & 79 & 84 & 92 & 108 & 103 & 109 & 117 & 97,697 & $93.88 \%$ \\
\hline & 101 & 90 & 78 & 118 & 100 & 114 & 112 & 105 & 153,978 & $99.15 \%$ \\
\hline & 120 & 120 & 120 & 120 & 120 & 120 & 120 & 120 & 259,128 & $100.00 \%$ \\
\hline \multirow{6}{*}{ Scenario 2} & 88 & 81 & 85 & 96 & 110 & 104 & 111 & 106 & 90,548 & $93.96 \%$ \\
\hline & 90 & 86 & 106 & 119 & 120 & 113 & 116 & 116 & 141,450 & $99.10 \%$ \\
\hline & 120 & 120 & 120 & 120 & 120 & 120 & 120 & 120 & 229,177 & $100.00 \%$ \\
\hline & \multicolumn{10}{|c|}{ Expected weekly demand of Scenarios 3 and 4} \\
\hline & 76 & 95 & 113 & 139 & 107 & 88 & 69 & 88 & & \\
\hline & \multicolumn{8}{|c|}{ Average number of jobs released each week } & $\widehat{E}[T C(\mathbf{x})]$ & $\widehat{\mathrm{E}}[F R(\mathbf{x})]$ \\
\hline \multirow{3}{*}{ Scenario 3} & 88 & 118 & 115 & 119 & 109 & 110 & 76 & 83 & 112,235 & $94.85 \%$ \\
\hline & 116 & 117 & 119 & 120 & 112 & 117 & 96 & 119 & 159,374 & $99.24 \%$ \\
\hline & 120 & 120 & 120 & 120 & 120 & 120 & 120 & 120 & 178,017 & $99.51 \%$ \\
\hline \multirow{3}{*}{ Scenario 4} & 101 & 118 & 114 & 114 & 111 & 114 & 86 & 96 & 98,539 & $94.33 \%$ \\
\hline & 117 & 120 & 120 & 115 & 115 & 120 & 99 & 102 & 132,431 & $98.24 \%$ \\
\hline & 120 & 120 & 120 & 120 & 120 & 120 & 120 & 120 & 146,337 & $98.77 \%$ \\
\hline
\end{tabular}

\section{Summary}

This work developed a metamodel-based Monte Carlo simulation (MCS) method to address a fundamental issue for optimization of production planning: the quantification of the relationships between a release plan of jobs and its resulting performance metrics (total cost and fill rate), which serves as the basis to find Pareto-optimal release plans. The MCS method is able to overcome the lack of fidelity of analytical methods and the computational burden of DES, allows for rapid and accurate evaluation of a release plan in terms of its performance metrics, which include not only the expectations (e.g., the mean cost) but also the variances (e.g., the variance of the cost) and probabilities of interest (e.g., the fill rate). The evaluation time required by the MCS is independent of the complexity of the real systems being investigated.

The real-time "what if" analysis rendered by the MCS method provides the necessary basis for the optimization of production planning in a timely manner. The MCS-based multi-objective optimization problem has been solved for the production planning of a scaleddown semiconductor fabrication system. The evaluation quality of the MCS is demonstrated by comparing the MCS estimates with those given by DES. The non-dominated solutions 
obtained from the multi-objective optimization allow decision makers to weigh the trade-offs between conflicting objectives such as minimizing the expected total cost and maximizing the fill rate.

\section{Acknowledgements}

This work was supported by National Science Foundation Grant No. CMMI-1068131. Any opinions, findings, and conclusions or recommendations expressed in this material are those of the authors and do not necessarily reflect the views of the National Science Foundation.

\section{Appendix A. Configuration of the Example System}

The system considered in the empirical study is a scaled-down semiconductor fabrication system described in [92]. It consists of 9 workstations, and includes the major features of real semiconductor fabrication system [92] such as re-entrant flows, machine failures, and batch processing. One job type is considered in this work with job processing sequence shown in Figure A.6. Each job has 14 processing steps, and needs to visit workstations 1, 4 and 6 multiple times. The inter-arrival time of jobs are assumed to follow an exponential distribution with arrival rate specified by the job release plan. Table A.4 gives the specific configuration of each of the 9 workstations. The first three rows of the table specify for each station the number of machines, batch processing size, and whether the machines are subject to random failures. Lognormal distributions are assumed for all the processing times (PT), and Weibull distributions for all the times to failure (TTF) and times to repair (TTR). For each station, the means and standard deviations (Stdev) of PT, TTF and TTR are also provided in Table A.4. The scale-down system is subject to substantial variability caused by machine failures and random processing times.

Table A.4: Configuration of workstations.

\begin{tabular}{lccccccccc}
\hline Station Index & 1 & 2 & 3 & 4 & 5 & 6 & 7 & 8 & 9 \\
\hline Number of Machines & 1 & 1 & 1 & 2 & 1 & 1 & 1 & 1 & 1 \\
Batch Size (Min/Max) & $2 / 4$ & $2 / 4$ & 1 & 1 & 1 & 1 & 1 & 1 & 1 \\
Failure & No & No & Yes & No & Yes & Yes & Yes & Yes & No \\
Mean of PT (minutes) & 80 & 220 & 80 & 40 & 25 & 22 & 40 & 50 & 50 \\
Stdev of PT(minutes) & 7 & 16 & 7 & 4 & 2 & 2.4 & 4 & 4 & 5 \\
Mean of TTF (minutes) & - & - & 720 & - & 1100 & 1170 & 720 & 1333 & - \\
Stdev of TTF (minutes) & - & - & 720 & - & 1100 & 1170 & 720 & 1333 & - \\
Mean of TTR (minutes) & - & - & 108.3 & - & 117.4 & 126.4 & 108.3 & 180.5 & - \\
Stdev of TTR (minutes) & - & - & 73.6 & - & 79.7 & 85.8 & 73.6 & 122.6 & - \\
\hline
\end{tabular}




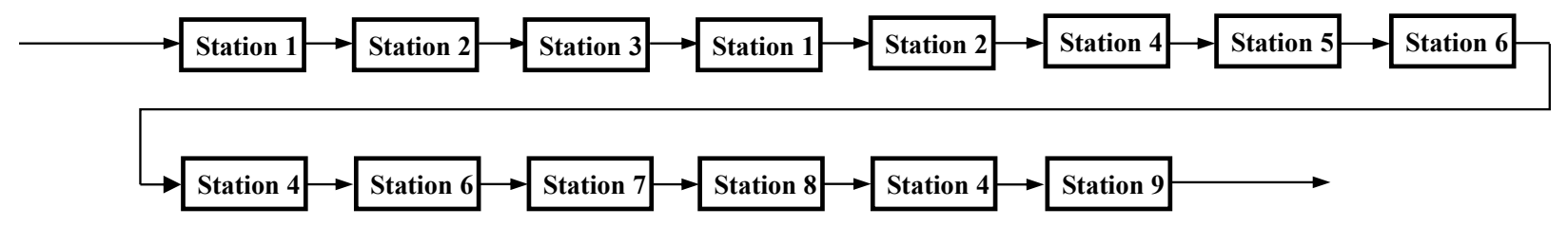

Figure A.6: Job processing sequence.

\section{Appendix B. Estimation and Evaluation of the Metamodel}

The metamodeling method (Section 4) was applied to the system described in Appendix A to obtain the fitted metamodel. DES was carried out to collect the estimation data set (EDS). Following the experimental design strategies in [54, the input arrival rate function $x(t)$ is specified as in Figure B.7a for the EDS. $x(t)$ is a piecewise constant function with 5 distinct levels corresponding to five system utilizations: $[0.71,0.50,0.92,0.61,0.82]$. The length of a DES replication is $L=1260$ time units with a total of $R=75000$ replications. The collected EDS takes the form of (3) and is used to fit the metamodel following Section 5.1 of [54]. The fitted metamodel is given in (B.1)-(B.9).

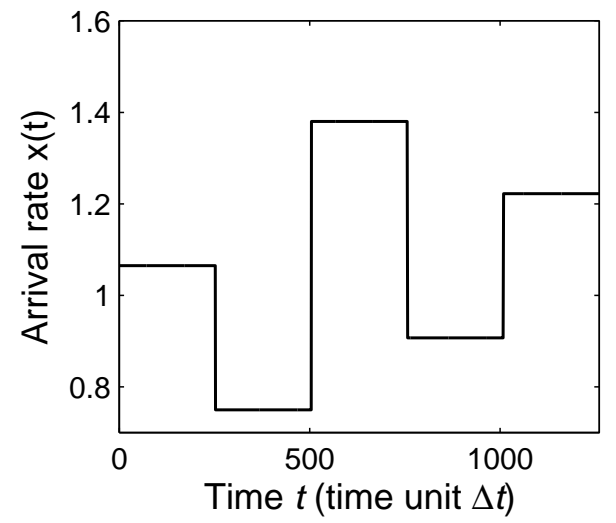

(a) EDS

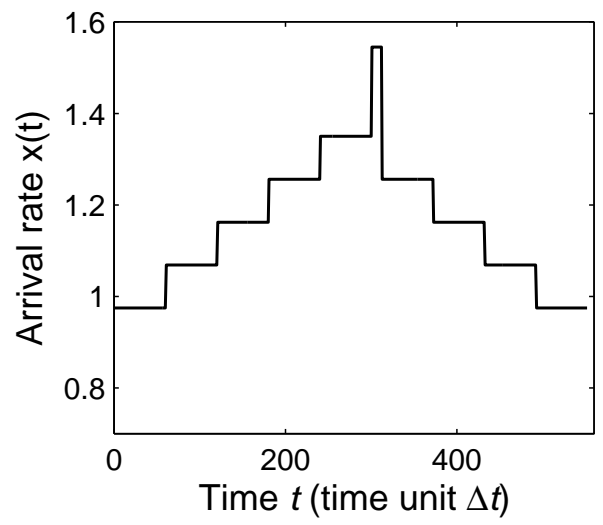

(c) VDS 2

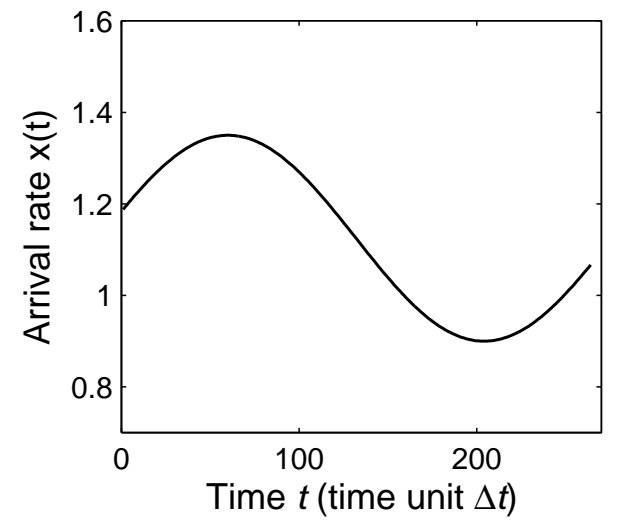

(b) VDS 1

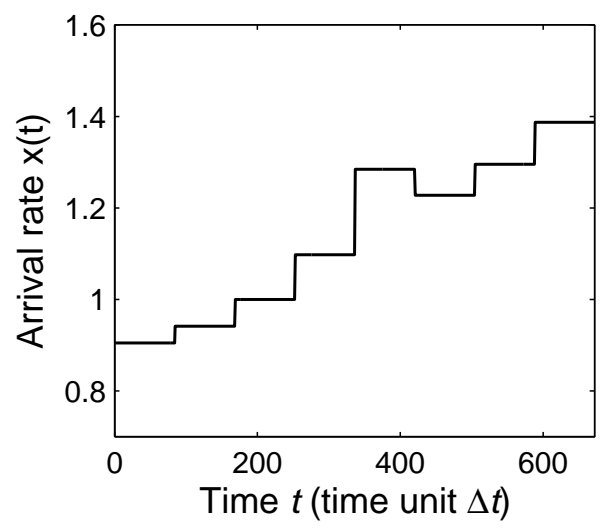

(d) VDS 3

Figure B.7: Input arrival rate functions for the estimation data set (EDS) and three validation data sets (VDS). 


$$
\begin{aligned}
\widehat{m}_{1}(t)= & m_{1}(t-1)-d_{1}(t-1)+x(t-1) \\
\widehat{d}_{1}(t)= & 0.0179+0.0434 m_{1}(t-1)+0.4561 d_{1}(t-1)-0.0066 m_{2}(t-1) \\
& +0.0071 m_{12}(t-1)-0.1449 d_{11}(t-1)-0.0093 m_{1}(t-1) d_{2}(t-1) \\
& -0.0034 m_{1}(t-1) d_{11}(t-1)+0.4666 d_{1}(t-1)^{2}+0.0072 d_{1}(t-1) m_{2}(t-1) \\
& -0.0082 d_{1}(t-1) m_{11}(t-1)+0.0004 m_{2}(t-1) d_{11}(t-1)+0.0741 d_{2}(t-1) x(t-1) \\
& -0.0909 d_{12}(t-1) x(t-1)-0.0307 x(t-1)^{2}
\end{aligned}
$$

$$
\begin{aligned}
\widehat{m}_{2}(t)= & 0.5662-0.1149 m_{1}(t-1)-0.7456 d_{1}(t-1)+1.0536 m_{2}(t-1) \\
& -0.1939 m_{11}(t-1)+0.1469 m_{12}(t-1)-2.0810 e_{Q D}(t-1)+2.7935 x(t-1) \\
& +0.0119 m_{1}(t-1)^{2}+1.8148 m_{1}(t-1) x(t-1)+1.0695 d_{1}(t-1) d_{2}(t-1) \\
& -1.2257 d_{1}(t-1) x(t-1)+0.0024 m_{2}(t-1) e_{Q D}(t-1)-0.0026 m_{12}(t-1) e_{Q D}(t-1) \\
& -0.5545 d_{11}(t-1) x(t-1)+1.3218 x(t-1)^{2}
\end{aligned}
$$

$$
\begin{aligned}
\widehat{d}_{2}(t)= & 0.0875+0.0278 m_{1}(t-1)+0.7497 d_{1}(t-1)+0.0304 m_{1} d_{1}(t-1) \\
& -0.0155 m_{1}(t-1) d_{1}(t-1)-0.0215 m_{1}(t-1) d_{11}(t-1)+0.3201 d_{1}(t-1)^{2} \\
& +0.2443 d_{1}(t-1) d_{2}(t-1)+0.1496 d_{2}(t-1) x(t-1)-0.1550 d_{12}(t-1) x(t-1) \\
& -0.1007 x(t-1)^{2}
\end{aligned}
$$

$$
\begin{aligned}
\widehat{m}_{11}(t)= & 0.1792-0.0852 m_{1}(t-1)+0.2214 d_{1}(t-1)+1.0823 m_{2}(t-1) \\
& -0.1283 m_{11}(t-1)+0.0465 m_{12}(t-1)-0.9557 e_{Q D}(t-1)+0.0044 m_{1}(t-1)^{2} \\
& 0.9168 m_{1}(t-1) x(t-1)-0.00003 m_{12}(t-1) e_{Q D}(t-1)+0.3236 x(t-1)^{2}
\end{aligned}
$$

.




$$
\begin{aligned}
& +1.0271 m_{1}(t-1) x(t-1)-0.3062 d_{1}(t-1) d_{12}(t-1)+0.0124 d_{1}(t-1) e_{Q D}(t-1) \\
& -0.0018 m_{2}(t-1)^{2}+0.0035 m_{2}(t-1) m_{11}(t-1)+0.2436 d_{2}(t-1) x(t-1) \\
& -0.0017 m_{11}(t-1)^{2}-0.0001 m_{12}(t-1) e_{Q D}(t-1)-0.5429 x(t-1)^{2}
\end{aligned}
$$

$$
\begin{aligned}
\widehat{d}_{11}(t)= & -0.0916-0.0294 m_{1}(t-1)+0.4253 d_{1}(t-1)-0.0015 m_{2}(t-1) \\
& +0.0886 e_{Q D}(t-1)+0.0330 m_{1}(t-1) d_{1}(t-1)-0.0101 m_{1}(t-1) d_{2}(t-1) \\
& +0.1408 d_{1}(t-1) x(t-1)+0.0080 m_{2}(t-1) x(t-1)+0.1928 d_{2}(t-1) d_{11}(t-1) \\
& -0.0153 m_{11}(t-1) x(t-1)+0.0083 m_{12}(t-1) x(t-1)-0.0173 d_{12}(t-1) e_{Q D}(t-1) \\
& -0.0261 e_{Q D}(t-1) x(t-1)+x(t-1)^{2}
\end{aligned}
$$

$$
\begin{aligned}
\widehat{d}_{12}(t)= & -0.1667+0.0103 m_{1}(t-1)+0.2946 d_{1}(t-1)-0.0116 m_{2}(t-1) \\
& +0.0105 m_{12}(t-1)+0.0699 e_{Q D}(t-1)-0.0050 m_{1}(t-1) d_{2}(t-1) \\
& +0.0081 m_{1}(t-1) x(t-1)+0.0051 d_{1}(t-1) m_{2}(t-1)-0.0062 d_{1}(t-1) m_{12}(t-1) \\
& +0.4627 d_{1}(t-1) d_{12}(t-1)+0.0011 m_{12}(t-1) d_{12}(t-1)-1.4809 d_{11}(t-1)^{2} \\
& +3.3272 d_{11}(t-1) d_{12}(t-1)-0.0605 d_{11}(t-1) x(t-1)-1.7835 d_{12}(t-1)^{2} \\
& -0.0285 d_{12}(t-1) e_{Q D}(t-1)
\end{aligned}
$$

$$
\begin{aligned}
\widehat{e}_{Q D}(t)= & -0.6245+0.1760 m_{1}(t-1)+0.0134 m_{12}(t-1)-2.4162 d_{12}(t-1) \\
& -0.2578 m_{1}(t-1) d_{1}(t-1)+0.8689 m_{1}(t-1) d_{11}(t-1)-0.4930 m_{1}(t-1) d_{12}(t-1) \\
& -0.1511 m_{1}(t-1) x(t-1)+6.2565 d_{1}(t-1) x(t-1)-0.0275 m_{11}(t-1) d_{11}(t-1) \\
& +0.0217 m_{12}(t-1) d_{11}(t-1)-9.5731 d_{11}(t-1) x(t-1)+0.7797 e_{Q D}(t-1) \\
& -0.1312 d_{12}(t-1) e_{Q D}(t-1)+6.1842 d_{12}(t-1) x(t-1)+0.9478 d_{12}^{2} \\
& -0.6269 x(t-1)^{2}
\end{aligned}
$$

To evaluate the prediction accuracy of the fitted metamodel, three validation data sets (VDS) are collected by running 70000 replications via DES using the input arrival rate functions shown in Figures B.7b B.7d, respectively. For VDS 1, $x(t)$ is a piecewise constant function which allows the system to be temporarily overloaded. For VDS 2, $x(t)$ is a sine wave function with the highest and lowest arrival rates correspond to system utilizations of 0.9 and 0.6 respectively. These two VDS are designed to test the metamodel's ability to predict both transient and steady-state behavior of the system. For VDS $3, x(t)$ is a non- 
dominated release plan obtained from the multi-objective optimization (Section 7.3 ): the first non-dominated solution for Scenario 1 given in Table 3. The release plan is converted to the number of arrivals per $\Delta t$, and plotted in Figure B.7d, with the 8 distinct levels being

$$
[0.9,0.95,1.01,1.10,1.29,1.23,1.29,1.40]
$$

corresponding to the system utilization levels of $[0.60,0.63,0.67,0.73,0.86,0.82,0.86,0.93]$.

The characteristics of system outputs estimated from the VDS serve as the "true" values and are compared with the metamodel-predicted results. As pointed out in Section 4.4 , the system's future outputs can be recursively computed by feeding the arrival rate (of a VDS) to the metamodel, and obtained within a second. The comparison results are shown in Figures B.8 B.10. The metamodel prediction, which is represented by the solid curves, is able to accurately track the "true" values, which are plotted as the dashed curves. 


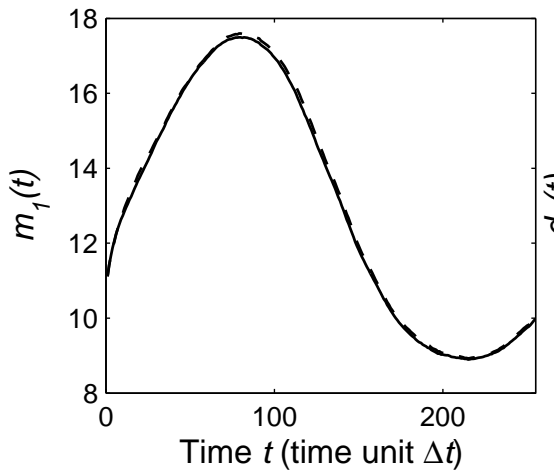

(a) $m_{1}(t)$

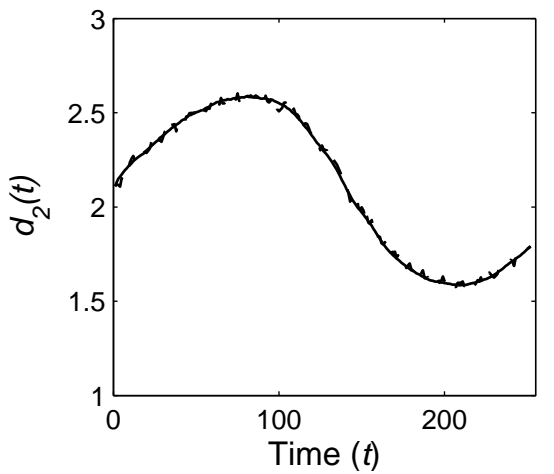

(d) $d_{2}(t)$

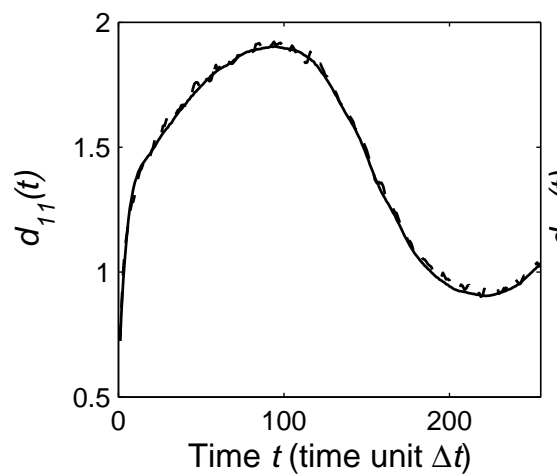

(g) $d_{11}(t)$

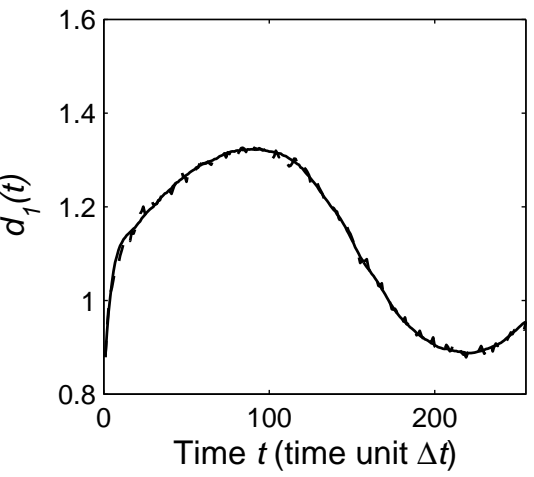

(b) $d_{1}(t)$

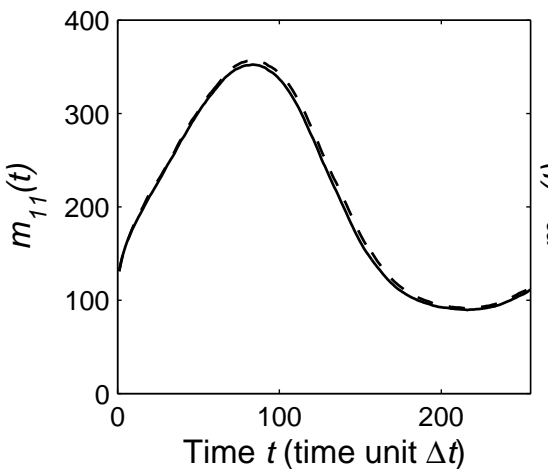

(e) $m_{11}(t)$

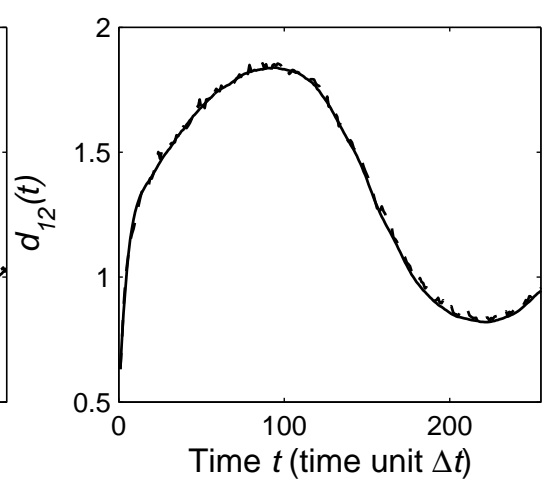

(h) $d_{12}(t)$

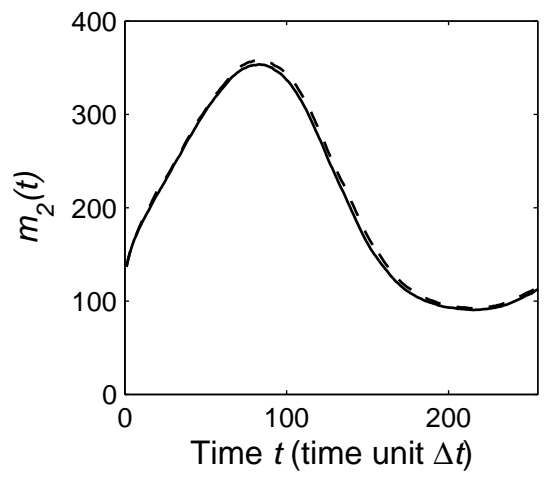

(c) $m_{2}(t)$

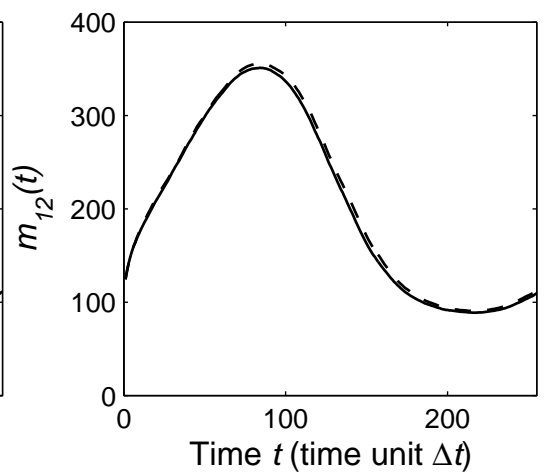

(f) $m_{12}(t)$

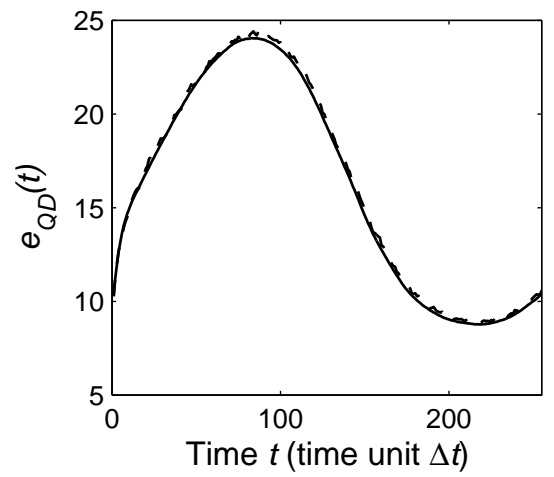

(i) $e_{Q D}(t)$

Figure B.8: Evaluation of the fitted metamodel using VDS 1: dashed curves denote the "true" values and solid curves represent the metamodel-predicted results. 


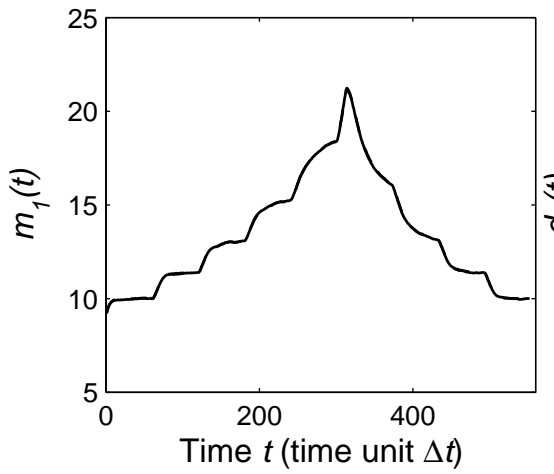

(a) $m_{1}(t)$

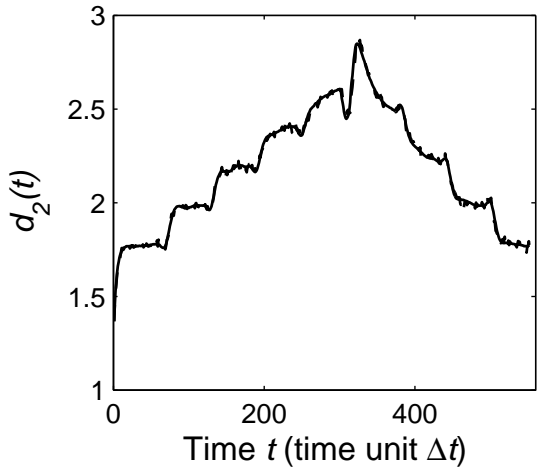

(d) $d_{2}(t)$

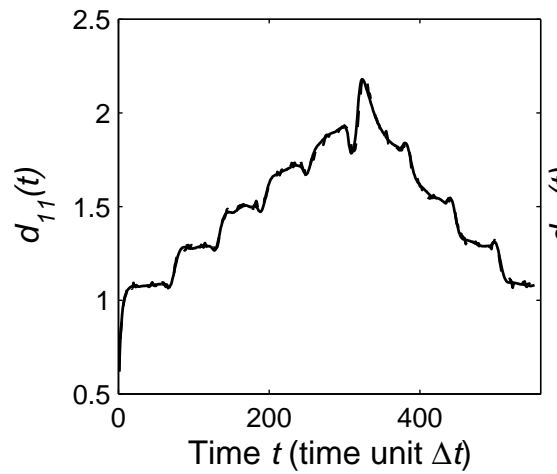

(g) $d_{11}(t)$

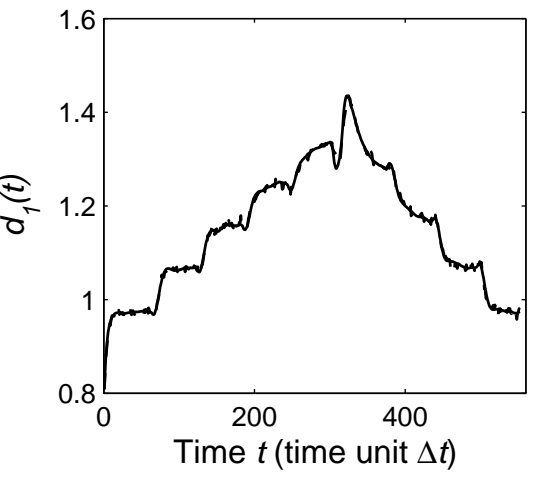

(b) $d_{1}(t)$

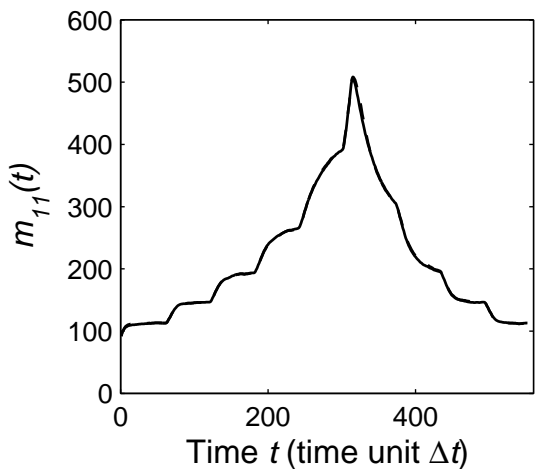

(e) $m_{11}(t)$

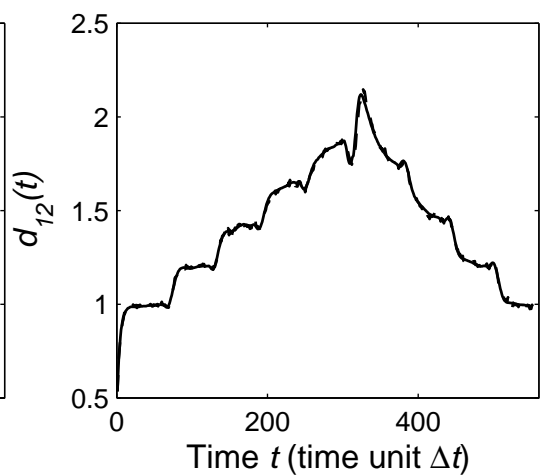

(h) $d_{12}(t)$

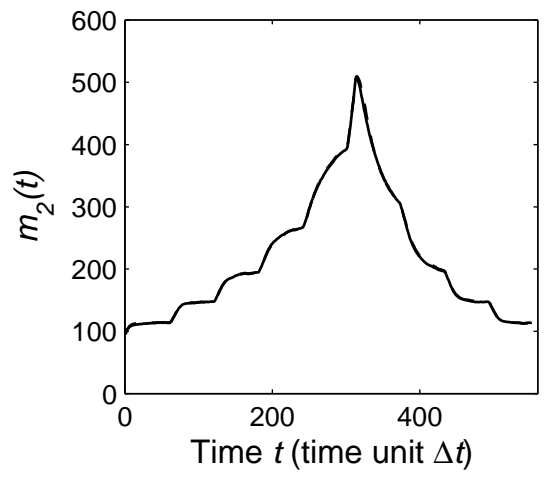

(c) $m_{2}(t)$

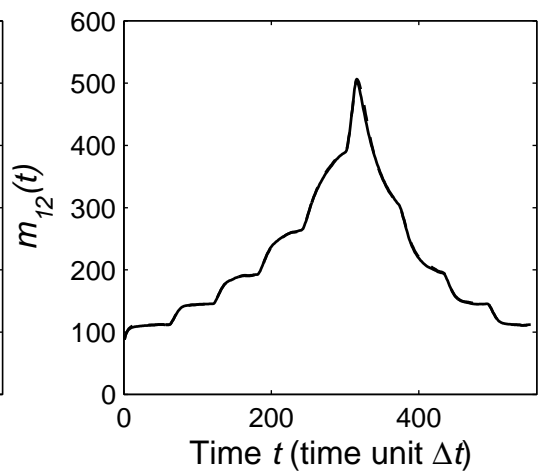

(f) $m_{12}(t)$

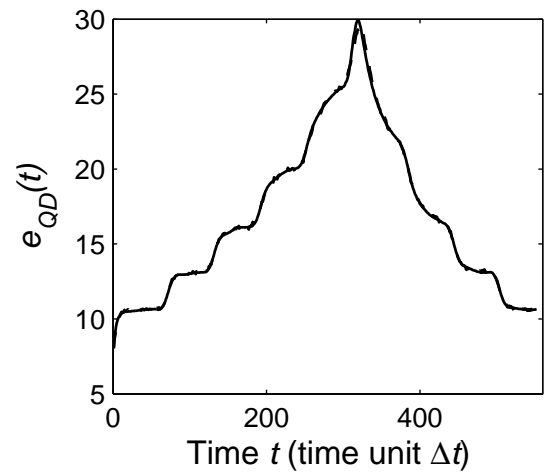

(i) $e_{Q D}(t)$

Figure B.9: Evaluation of the fitted metamodel using VDS 2: dashed curves denote the "true" values and solid curves represent the metamodel-predicted results. 


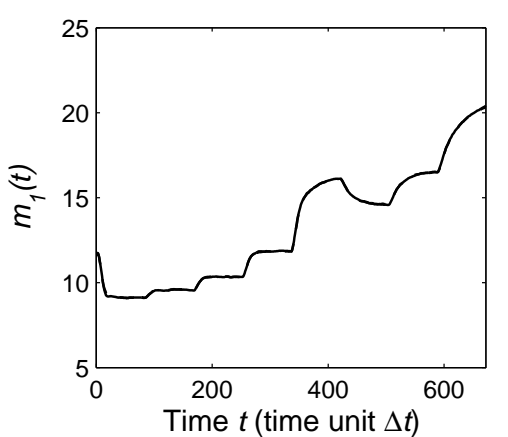

(a) $m_{1}(t)$

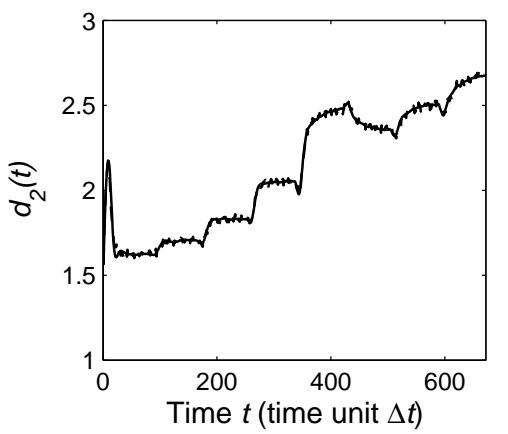

(d) $d_{2}(t)$

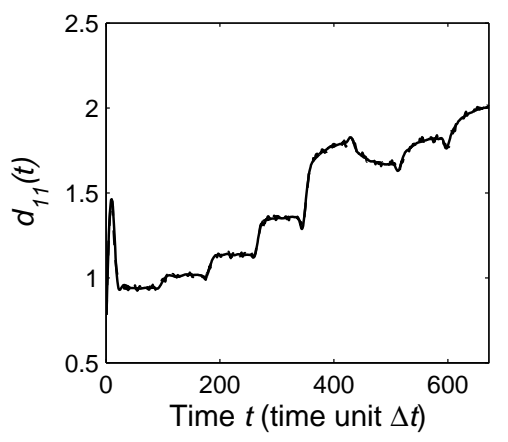

(g) $d_{11}(t)$

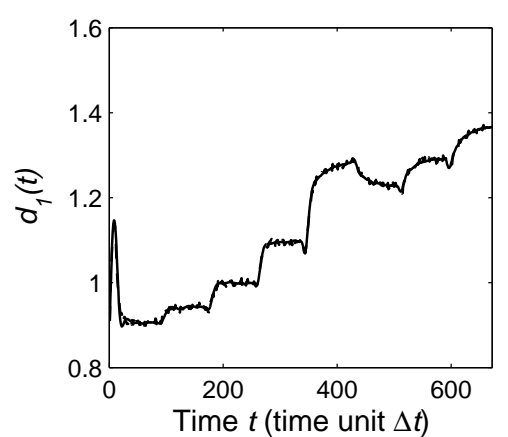

(b) $d_{1}(t)$

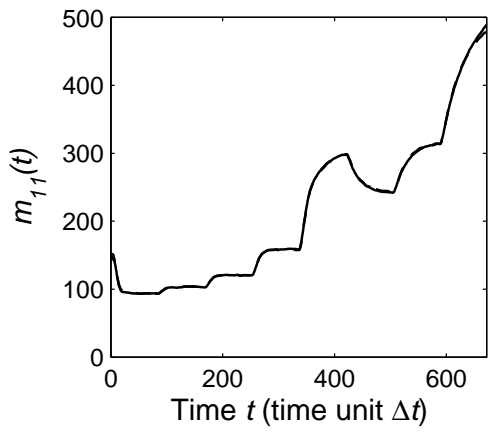

(e) $m_{11}(t)$

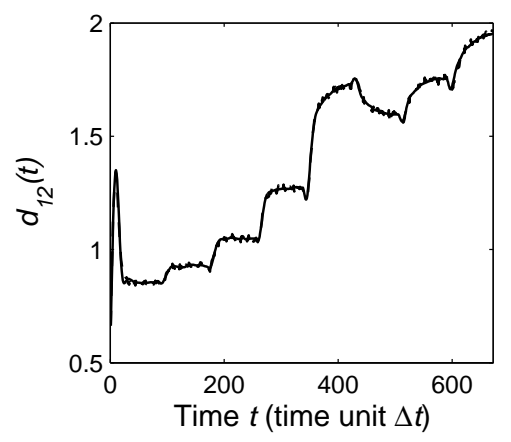

(h) $d_{12}(t)$

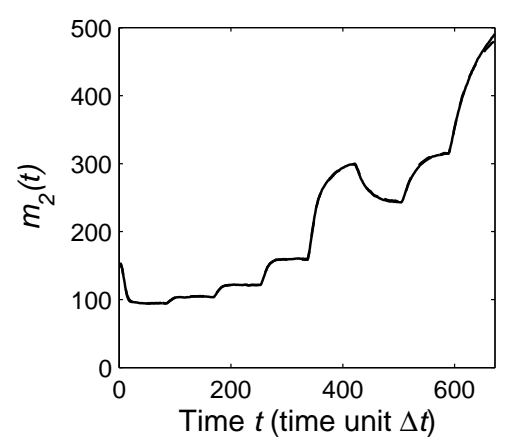

(c) $m_{2}(t)$

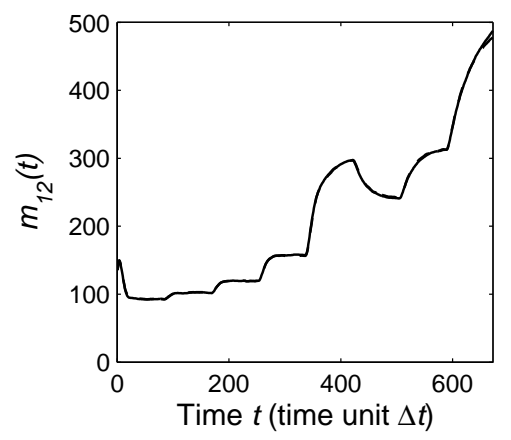

(f) $m_{12}(t)$

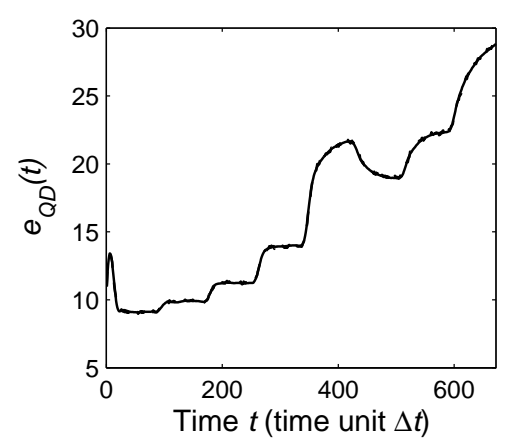

(i) $e_{Q D}(t)$

Figure B.10: Evaluation of the fitted metamodel using VDS 3: dashed curves denote the "true" values and solid curves represent the metamodel-predicted results. 


\section{Appendix C. Yule-Walker Estimator of Autoregressive Processes}

Let $Z(t)$ be an autoregressive process representing one of the two stationary processes from steady-state simulation: WIP $Q(t)$ and departures $D(t)$. The stationary process $Z(t)$ can be written in the autoregressive form

$$
Z(t)=\sum_{j=1}^{J} \alpha_{j} Z(t-j)+\varepsilon(t) ; \quad t=1,2, \ldots, N
$$

where $J$ denotes the autoregressive order, $\left\{\alpha_{j} ; j=1,2, \ldots, J\right\}$ the autoregressive coefficients, $\varepsilon(t)$ the innovation term, and $N$ the number of observations.

The model selection in Section 5.1 .2 between N-INAR and N-SINAR is made by performing the following hypothesis test:

$$
H_{0}: \alpha_{j} \geq 0 ; \text { vs. } H_{a}: \alpha_{j}<0
$$

for $j=1,2, \ldots, J$. If $H_{0}$ is rejected for any $j$, then N-SINAR will be selected; otherwise, N-INAR will be adopted. Yule-Walker estimation and inference are performed in this work to test the hypothesis (C.1), based on the steady-state DES data $\left\{Z_{r}(t) ; t=1,2 \ldots, N ; r=\right.$ $1,2, \ldots, R\}$, where $R$ is the number of replications. Let

$$
\widetilde{Z}(t)=\frac{1}{R} \sum_{r=1}^{R} Z_{r}(t)
$$

By the Central Limit Theorem, $\widetilde{Z}(t)$ approximately follows a normal distribution with

$$
\begin{array}{cl}
\mathrm{E}[\widetilde{Z}(t)]=\mathrm{E}[Z(t)], & \operatorname{Var}[\widetilde{Z}(t)]=\frac{1}{R} \operatorname{Var}[Z(t)], \\
\operatorname{Cov}[\widetilde{Z}(t+j), \widetilde{Z}(t)]=\frac{1}{R} \operatorname{Cov}[Z(t+j), Z(t)], & \operatorname{Corr}[\widetilde{Z}(t+j), \widetilde{Z}(t)]=\operatorname{Corr}[Z(t+j), Z(t)]
\end{array}
$$

when $R$ is large. Hence, $\widetilde{Z}(t)$ can be modeled by a $\operatorname{AR}(J)$ process

$$
\widetilde{Z}(t)=\sum_{j=1}^{J} \alpha_{j} \widetilde{Z}(t-j)+\varepsilon(t) ; \quad t=1,2, \ldots, N
$$

where $\varepsilon(t)$ is the i.i.d normally-distributed innovation term with $\operatorname{Var}[\varepsilon(t)]=\sigma^{2}$. Denote

$$
\gamma_{0}=\operatorname{Var}[\widetilde{Z}(t)], \quad \operatorname{Cov}[\widetilde{Z}(t+j), \widetilde{Z}(t)]=\gamma_{j}
$$

the Yule-Walker equation [57, 88] is written as

$$
\Gamma \alpha=\gamma
$$


where

$$
\boldsymbol{\Gamma}=\left(\begin{array}{ccccc}
\gamma_{0} & \gamma_{1} & \gamma_{2} & \cdots & \gamma_{J-1} \\
\gamma_{1} & \gamma_{0} & \gamma_{1} & \cdots & \gamma_{J-2} \\
\vdots & \vdots & \vdots & \cdots & \vdots \\
\gamma_{J-1} & \gamma_{J-2} & \gamma_{J-3} & \cdots & \gamma_{0}
\end{array}\right), \quad \boldsymbol{\alpha}=\left(\begin{array}{c}
\alpha_{1} \\
\alpha_{2} \\
\vdots \\
\alpha_{J}
\end{array}\right), \quad \boldsymbol{\gamma}=\left(\begin{array}{c}
\gamma_{1} \\
\gamma_{2} \\
\vdots \\
\gamma_{J}
\end{array}\right)
$$

Replacing $\left\{\gamma_{0}, \gamma_{1}, \ldots, \gamma_{J}\right\}$ by the sample estimates $\left\{\widehat{\gamma}_{0}, \widehat{\gamma}_{1}, \ldots, \widehat{\gamma}_{J}\right\}$ in C.2, $\boldsymbol{\alpha}$ and $\sigma^{2}$ can be estimated as

$$
\widehat{\boldsymbol{\alpha}}=\widehat{\boldsymbol{\Gamma}}^{-1} \widehat{\boldsymbol{\gamma}}, \quad \widehat{\sigma}^{2}=\widehat{\gamma}_{0}-\widehat{\boldsymbol{\alpha}}^{\top} \widehat{\boldsymbol{\gamma}}
$$

Since $Z(t)$ and $\widetilde{Z}(t)$ have the same autocorrelation structure, it can be shown that these two processes have the same Yule-Walker estimates for $\boldsymbol{\alpha}$ [61, 67]. The hypothesis (C.1) is performed based on $\widetilde{Z}(t)$.

When the sample size $N$ is large, the Yule-Walker estimator $\widehat{\boldsymbol{\alpha}}$ of $\widetilde{Z}(t)$ follows the asymptotic normal distribution [88, 99

$$
\widehat{\boldsymbol{\alpha}} \sim \mathcal{N}\left(\boldsymbol{\alpha}, N^{-1} \sigma^{2} \boldsymbol{\Gamma}^{-1}\right)
$$

and

$$
\frac{\widehat{\alpha}_{j}-\alpha_{j}}{\sqrt{\widehat{w}_{j j} / N}} \sim \mathcal{N}(0,1)
$$

where $\widehat{w}_{j j}$ denotes the $j^{\text {th }}$ diagonal element of $\widehat{\sigma}^{2} \widehat{\boldsymbol{\Gamma}}^{-1}$. Define $\Phi$ as the the cumulative distribution function of the standard normal distribution. For the hypothesis test (C.1) with significance level $\delta$, if $\frac{\widehat{\alpha}_{j}}{\sqrt{\widehat{w}_{j j} / N}}$ is less than $\Phi^{-1}(\delta), H_{0}$ will be rejected; otherwise, $H_{0}$ is considered acceptable.

\section{Appendix D. Model Fitting of Bivariate Time Series}

For the bivariate output process $(Q(t), D(t))^{\top}$ driven by an arrival process, the fitted metamodel is able to predict its characteristics

$$
\widehat{\mathbf{y}}(t)=\left(\widehat{m}_{1}(t), \widehat{m}_{2}(t), \widehat{d}_{1}(t), \widehat{d}_{2}(t), \widehat{m}_{11}(t), \ldots, \widehat{m}_{1 J_{Q}}(t), \widehat{d}_{11}(t), \ldots, \widehat{d}_{1 J_{D}}(t), \widehat{e}_{Q D}(t)\right)^{\top}
$$

Based on $\widehat{\mathbf{y}}(t)$, how to fit a bivariate time-series model for $(Q(t), D(t))^{\top}$, with the identified time-series model family (Section 5.1.3)?

\section{Appendix D.1. Properties of Autoregressive Models}

The general form of the candidate autoregressive models (4) identified for the output processes is re-written below for convenience.

$$
Z(t)=\sum_{j=1}^{J} \mathcal{R}\left(\alpha_{j}(t), Z(t-j)\right)+\varepsilon(t) ; \quad t=1,2, \ldots
$$


As discussed in Section 5.1.1, the random operator $\mathcal{R}$ can be scalar multiplication, binomial thinning "o" or generalized thinning " $F(\cdot) \circ$ ", corresponding to N-AR, N-INAR and NSINAR model respectively. A time series $Z(t)$ following any of the three models has the following properties [57]62] 61] 67]:

$$
\begin{aligned}
& \mathrm{E}\left[\mathcal{R}\left(\alpha_{j}(t), Z(t-j)\right)\right]=\alpha_{j}(t) \mathrm{E}[Z(t-j)] \\
& \mathrm{E}\left[\mathcal{R}\left(\alpha_{j}(t), Z(t-j)\right)^{2}\right]=\left(\alpha_{j}(t)\right)^{2} \mathrm{E}\left[(Z(t-j))^{2}\right]+f_{\mathcal{R}}\left(\alpha_{j}(t), \mathrm{E}[Z(t-j)]\right) \\
& \operatorname{Var}\left[\mathcal{R}\left(\alpha_{j}(t), Z(t-j)\right)\right]=\left(\alpha_{j}(t)\right)^{2} \operatorname{Var}[Z(t-j)]+f_{\mathcal{R}}\left(\alpha_{j}(t), \mathrm{E}(Z(t-j))\right) \\
& \mathrm{E}\left[\mathcal{R}\left(\alpha_{j}(t), Z(t-j)\right), \mathcal{R}\left(\alpha_{i}(t), Z(t-i)\right)\right]=\alpha_{j}(t) \alpha_{i}(t) \mathrm{E}[Z(t-j) Z(t-i)] \\
& \operatorname{Cov}\left[\mathcal{R}\left(\alpha_{j}(t), Z(t-j)\right), \mathcal{R}\left(\alpha_{i}(t), Z(t-i)\right)\right]=\alpha_{j}(t) \alpha_{i}(t) \operatorname{Cov}[Z(t-j), Z(t-i)]
\end{aligned}
$$

The form of $f_{\mathcal{R}}(\cdot)$ in (D.4) and (D.5) is model-dependent: For N-AR, $f_{\mathcal{R}}\left(\alpha_{j}(t), \mathrm{E}[Z(t-\right.$ $j)])=0$; for N-INAR, $f_{\mathcal{R}}\left(\alpha_{j}(t), \mathrm{E}[Z-j]\right)=\alpha_{j}(t)\left(1-\alpha_{j}(t)\right) \mathrm{E}[Z(t-j)]$; and for N-SINAR, $f_{\mathcal{R}}\left(\alpha_{j}(t), \mathrm{E}[Z(t-j)]\right)=\left(1-\alpha_{j}(t)^{2}\right) \mathrm{E}[Z(t-j)]$.

Based on (D.3)-D.7), some properties of the time series $Z(t)$ and the innovation process $\varepsilon(t)$ can be derived as follows. Specifically, we have

$$
\begin{aligned}
\operatorname{Cov}(Z(t), Z(t-j)) & =\sum_{i=1}^{J} \operatorname{Cov}\left[\mathcal{R}\left(\alpha_{i}(t), Z(t-i)\right), Z(t-j)\right]+\operatorname{Cov}[\varepsilon(t), Z(t-j)] \\
& =\sum_{i=1}^{J} \alpha_{i}(t) \operatorname{Cov}[Z(t-i), Z(t-j)] .
\end{aligned}
$$

Step (D.8) is obtained by expressing $Z(t)$ and $Z(t-j)$ in terms of (D.2). Step (D.9) employs the property (D.7) and the assumption that $Z(t-j)$ is independent of $\varepsilon(t)$.

By taking expectation and variance on both sides of (D.2) and using the properties (D.3) and (D.5), the mean and variance of $\varepsilon(t)$ can be expressed in terms of the characteristics of $Z(t)$ as follows:

$$
\begin{aligned}
\mathrm{E}[\varepsilon(t)]= & \mathrm{E}[Z(t)]-\sum_{i=1}^{J} \alpha_{i}(t) \mathrm{E}[Z(t-i)] \\
\operatorname{Var}[\varepsilon(t)]= & \operatorname{Var}[Z(t)]-\sum_{i=1}^{J} \alpha_{i}^{2}(t) \operatorname{Var}[Z(t-i)]-\sum_{i=1}^{J} f_{\mathcal{R}}\left(\alpha_{i}(t), \mathrm{E}[Z(t-i)]\right) \\
& -2 \sum_{i=1}^{J-1} \sum_{j=i+1}^{J} \alpha_{i}(t) \alpha_{j}(t) \operatorname{Cov}[Z(t-i), Z(t-j)] .
\end{aligned}
$$

As will be seen below, the properties (D.9) - D.11) enable the derivation from the metamodelpredicted characteristics (D.1) to the fitted parameters that specify the bivariate time-series model. 


\section{Appendix D.2. Fitting the Bivariate Time-Series Model}

The following expectations, variances and covariances can be easily obtained from the metamodel prediction (D.1):

$$
\begin{aligned}
\widehat{\mathrm{E}}[\widehat{Q}(t)] & =\widehat{m}_{1}(t) \\
\widehat{\operatorname{Var}}[\widehat{Q}(t)] & =\widehat{m}_{2}(t)-\left(\widehat{m}_{1}(t)\right)^{2} \\
\widehat{\operatorname{Cov}}[\widehat{Q}(t), \widehat{Q}(t-j)] & =\widehat{m}_{1 j}(t)-\widehat{m}_{1}(t) \widehat{m}_{1}(t-j) \text { for } j=1,2, \ldots, J_{Q} \\
\widehat{\mathrm{E}}[\widehat{D}(t)] & =\widehat{d}_{1}(t) \\
\widehat{\operatorname{Var}}[\widehat{D}(t)] & =\widehat{d}_{2}(t)-\left(\widehat{d}_{1}(t)\right)^{2} \\
\widehat{\operatorname{Cov}}[\widehat{D}(t), \widehat{D}(t-j)] & =\widehat{d}_{1 j}(t)-\widehat{d}_{1}(t) \widehat{d}_{1}(t-j) \text { for } j=1,2, \ldots, J_{D} \\
\widehat{\operatorname{Cov}}[\widehat{Q}(t), \widehat{D}(t)] & =\widehat{e}_{Q D}(t)-\widehat{m}_{1}(t) \widehat{d}_{1}(t)
\end{aligned}
$$

with $t=1,2, \ldots, H$.

Following the procedures in [57, 61], we use (D.12) $(\mathrm{D} .18)$ to derive as follows the fitted model parameters for the bivariate time series: $\left\{\widehat{\alpha}_{j}^{(D)}(t) ; j=1,2, \ldots, J_{D}\right\}, \widehat{\mathrm{E}}\left[\widehat{\varepsilon}^{(Q)}(t)\right]$, $\widehat{\mathrm{E}}\left[\widehat{\varepsilon}^{(D)}(t)\right], \widehat{\operatorname{Var}}\left[\widehat{\varepsilon}^{(Q)}(t)\right], \widehat{\operatorname{Var}}\left[\widehat{\varepsilon}^{(D)}(t)\right]$, and $\widehat{\operatorname{Cov}}\left[\widehat{\varepsilon}^{(Q)}(t), \widehat{\varepsilon}^{(D)}(t)\right]$.

By employing $(\mathrm{D} .9),\left\{\widehat{\alpha}_{j}^{(Q)}(t) ; j=1,2, \ldots, J_{Q}\right\}, \widehat{\mathrm{E}}\left[\widehat{\varepsilon}^{(Q)}(t)\right]$ and $\widehat{\operatorname{Var}}\left[\widehat{\varepsilon}^{(Q)}(t)\right]$ are derived as follows:

$$
\widehat{\boldsymbol{\alpha}}^{(Q)}(t)=\left(\begin{array}{c}
\widehat{\alpha}_{1}^{(Q)}(t) \\
\widehat{\alpha}_{2}^{(Q)}(t) \\
\vdots \\
\widehat{\alpha}_{J_{Q}}^{(Q)}(t)
\end{array}\right)=\left(\widehat{\mathbf{A}}^{(Q)}(t)\right)^{-1} \widehat{\mathbf{b}}^{(Q)}(t),
$$

where $\widehat{\mathbf{A}}^{(Q)}(t)$ is a $J_{Q} \times J_{Q}$ matrix with the element in the $i^{\text {th }}$ row and $j^{\text {th }}$ column being:

$$
\widehat{\mathbf{A}}^{(Q)}(t)_{i j}=\widehat{\operatorname{Cov}}[\widehat{Q}(t-\min (i, j)), \widehat{Q}(t-\min (i, j)-|i-j|)], \quad 0<i, j \leq J_{Q},
$$

and

$$
\widehat{\mathbf{b}}^{(Q)}(t)=\left(\begin{array}{c}
\widehat{\operatorname{Cov}}[\widehat{Q}(t), \widehat{Q}(t-1)] \\
\widehat{\operatorname{Cov}}[\widehat{Q}(t), \widehat{Q}(t-2)] \\
\vdots \\
\widehat{\operatorname{Cov}}\left[\widehat{Q}(t), \widehat{Q}\left(t-J_{Q}\right)\right]
\end{array}\right) .
$$

Then, based on D.10-D.11), we have

$$
\widehat{\mathrm{E}}\left[\widehat{\varepsilon}^{(Q)}(t)\right]=\widehat{\mathrm{E}}[\widehat{Q}(t)]-\sum_{i=1}^{J_{Q}} \widehat{\alpha}_{i}^{(Q)}(t) \widehat{\mathrm{E}}[\widehat{Q}(t-i)]
$$




$$
\begin{aligned}
\widehat{\operatorname{Var}}\left[\widehat{\varepsilon}^{(Q)}(t)\right]= & \widehat{\operatorname{Var}}[\widehat{Q}(t)]-\sum_{i=1}^{J_{Q}}\left(\widehat{\alpha}_{i}^{(Q)}(t)\right)^{2} \widehat{\operatorname{Var}}[\widehat{Q}(t-i)] \\
& -\sum_{i=1}^{J_{Q}} f_{\mathcal{R}^{(Q)}}\left(\widehat{\alpha}_{i}^{(Q)}(t), \widehat{\mathrm{E}}[\widehat{Q}(t-i)]\right) \\
& -2 \sum_{i=1}^{J_{Q}} \sum_{j>i}^{J_{Q}} \widehat{\alpha}_{i}^{(Q)}(t) \widehat{\alpha}_{j}^{(Q)}(t) \widehat{\operatorname{Cov}}[\widehat{Q}(t-i), \widehat{Q}(t-j)]
\end{aligned}
$$

The remaining parameter to be estimated is $\widehat{\operatorname{Cov}}\left[\widehat{\varepsilon}^{(Q)}(t), \widehat{\varepsilon}^{(D)}(t)\right]$, which leads to the specified $\widehat{\operatorname{Cov}}[\widehat{Q}(t), \widehat{D}(t)]$ given by D.18). Assume $J_{Q} \geq J_{D}$ without loss of generality. The fitted bivariate model can be written as

$$
\left(\begin{array}{l}
\widehat{Q}(t) \\
\widehat{D}(t)
\end{array}\right)=\sum_{i=1}^{J_{Q}}\left(\begin{array}{c}
\mathcal{R}^{(Q)}\left(\widehat{\alpha}_{i}^{(Q)}(t), \widehat{Q}(t-i)\right) \\
\mathcal{R}^{(D)}\left(\widehat{\alpha}_{i}^{(D)}(t), \widehat{D}(t-i)\right)
\end{array}\right)+\left(\begin{array}{c}
\widehat{\varepsilon}^{(Q)}(t) \\
\widehat{\varepsilon}^{(D)}(t)
\end{array}\right)
$$

where $\widehat{\alpha}_{i}^{(D)}(t)=0$ for $i>J_{D}$. Denote

$$
\begin{gathered}
\widehat{\boldsymbol{\Sigma}}^{(Q D)}(t)=\widehat{\operatorname{Var}}\left[\left(\begin{array}{c}
\widehat{Q}(t) \\
\widehat{D}(t)
\end{array}\right)\right], \widehat{\boldsymbol{\Sigma}}^{(Q D)}\left(t_{1}, t_{2}\right)=\widehat{\operatorname{Cov}}\left[\left(\begin{array}{c}
\widehat{Q}\left(t_{1}\right) \\
\widehat{D}\left(t_{1}\right)
\end{array}\right),\left(\begin{array}{c}
\widehat{Q}\left(t_{2}\right) \\
\widehat{D}\left(t_{2}\right)
\end{array}\right)\right] ; t_{1} \neq t_{2}, \\
\widehat{\boldsymbol{\Sigma}}^{(\widehat{\boldsymbol{\varepsilon}})}(t)=\widehat{\operatorname{Var}}\left[\left(\begin{array}{l}
\widehat{\varepsilon}^{(Q)}(t) \\
\widehat{\boldsymbol{\varepsilon}}^{(D)}(t)
\end{array}\right)\right], \widehat{\boldsymbol{\Lambda}}_{i}(t)=\left(\begin{array}{cc}
\widehat{\alpha}_{i}^{(Q)}(t) & 0 \\
0 & \widehat{\alpha}_{i}^{(D)}(t)
\end{array}\right) .
\end{gathered}
$$

Based on the properties (D.4)-(D.5), it can be shown that

$$
\begin{aligned}
& \widehat{\operatorname{Var}}\left[\left(\begin{array}{c}
\mathcal{R}^{(Q)}\left(\widehat{\alpha}_{i}^{(Q)}(t), \widehat{Q}(t-i)\right) \\
\mathcal{R}^{(D)}\left(\widehat{\alpha}_{i}^{(D)}(t), \widehat{D}(t-i)\right)
\end{array}\right)\right]=\widehat{\boldsymbol{\Lambda}}_{i}(t) \widehat{\boldsymbol{\Sigma}}^{(Q D)}(t-i) \widehat{\boldsymbol{\Lambda}}_{i}(t)^{\top} \\
& +\left(\begin{array}{cc}
f_{\mathcal{R}^{(Q)}}\left(\widehat{\alpha}_{i}^{(Q)}(t), \widehat{E}[\widehat{Q}(t-i)]\right) & 0 \\
0 & f_{\mathcal{R}^{(D)}}\left(\widehat{\alpha}_{i}^{(D)}(t), \widehat{E}[\widehat{D}(t-i)]\right)
\end{array}\right) .
\end{aligned}
$$

Taking the variance on both sides of (D.22) and using (D.7), (D.23) and Lemma 2.1 in [62], we have

$$
\begin{aligned}
\widehat{\boldsymbol{\Sigma}}^{(\widehat{\boldsymbol{\varepsilon}})}(t) & =-\sum_{i=1}^{J_{Q}} \widehat{\boldsymbol{\Lambda}}_{i}(t) \widehat{\boldsymbol{\Sigma}}^{(Q D)}(t-i) \widehat{\boldsymbol{\Lambda}}_{i}(t)^{\top}-\sum_{i=1}^{J_{Q}} \sum_{j>i}^{J_{Q}} \widehat{\boldsymbol{\Lambda}}_{i}(t) \widehat{\boldsymbol{\Sigma}}^{(Q D)}(t-i, t-j) \widehat{\boldsymbol{\Lambda}}_{j}(t)^{\top} \\
& -\sum_{i=1}^{J_{Q}} \sum_{j>i}^{J_{Q}} \widehat{\boldsymbol{\Lambda}}_{j}(t) \widehat{\boldsymbol{\Sigma}}^{(Q D)}(t-j, t-i) \widehat{\boldsymbol{\Lambda}}_{i}(t)^{\top}+\widehat{\boldsymbol{\Sigma}}^{(Q D)}(t) \\
& -\sum_{i=1}^{J_{Q}}\left(\begin{array}{cc}
f_{\mathcal{R}^{(Q)}}\left(\widehat{\alpha}_{i}^{(Q)}(t), \widehat{E}[\widehat{Q}(t-i)]\right) & 0 \\
0 & f_{\mathcal{R}^{(D)}}\left(\widehat{\alpha}_{i}^{(D)}(t), \widehat{E}[\widehat{D}(t-i)]\right) .
\end{array}\right)
\end{aligned}
$$


Notice that $\widehat{\alpha}_{i}^{(D)}(t)=0$ for $i>J_{D}$, and $\widehat{\operatorname{Cov}}\left[\widehat{\varepsilon}^{(Q)}(t), \widehat{\varepsilon}^{(D)}(t)\right]$ is the off-diagonal element of $\widehat{\boldsymbol{\Sigma}}^{(\widehat{\boldsymbol{\varepsilon}})}(t)$ in D.24 and can be written as

$$
\begin{aligned}
\widehat{\operatorname{Cov}}\left[\widehat{\varepsilon}^{(Q)}(t), \widehat{\varepsilon}^{(D)}(t)\right]= & \widehat{\operatorname{Cov}}[\widehat{Q}(t), \widehat{Z}(t)]-\sum_{i=1}^{J_{D}} \widehat{\alpha}_{i}^{(Q)}(t) \widehat{\operatorname{Cov}}[\widehat{Q}(t-i), \widehat{D}(t-i)] \widehat{\alpha}_{i}^{(D)}(t) \\
& -\sum_{i=1}^{J_{D}} \sum_{j>i}^{J_{D}} \widehat{\alpha}_{i}^{(Q)}(t) \widehat{\operatorname{Cov}}[\widehat{Q}(t-i), \widehat{D}(t-j)] \widehat{\alpha}_{j}^{(D)}(t) \\
& -\sum_{i=1}^{J_{D}} \sum_{j>i}^{J_{D}} \widehat{\alpha}_{j}^{(Q)}(t) \widehat{\operatorname{Cov}}[\widehat{Q}(t-j), \widehat{D}(t-i)] \widehat{\alpha}_{i}^{(D)}(t) .
\end{aligned}
$$

In $(\mathrm{D} .25), \widehat{\operatorname{Cov}}[\widehat{Q}(t), \widehat{D}(t)]$ is a metamodel-predicted characteristics, and the remaining terms need to be estimated are

$$
\widehat{\operatorname{Cov}}[\widehat{Q}(t), \widehat{D}(t-i)], \widehat{\operatorname{Cov}}[\widehat{Q}(t-i), \widehat{D}(t)], \quad t=1,2, \ldots, H, \quad i=1,2, \ldots, J_{D}
$$

Using the properties D.3 - D.7), and the facts that $\widehat{Q}(t-j)$ is independent of $\widehat{\varepsilon}^{(Q)}(t)$ in 14 and $\widehat{D}(t-j)$ is independent of $\widehat{\varepsilon}^{(D)}(t)$ in 15$)$, the following results can be obtained:

$$
\begin{aligned}
\widehat{\operatorname{Cov}}[\widehat{Q}(t), \widehat{D}(t-i)] & =\widehat{\operatorname{Cov}}\left[\sum_{j=1}^{J_{Q}} \mathcal{R}^{(Q)}\left(\widehat{\alpha}_{j}^{(Q)}(t), \widehat{Q}(t-j)\right)+\widehat{\varepsilon}^{(Q)}(t), \widehat{D}(t-i)\right] \\
& =\sum_{j=1}^{J_{Q}} \widehat{\alpha}_{j}^{(Q)}(t) \widehat{\operatorname{Cov}}[\widehat{Q}(t-j), \widehat{D}(t-i)] \\
\widehat{\operatorname{Cov}}[\widehat{Q}(t-i), \widehat{D}(t)] & =\widehat{\operatorname{Cov}}\left[\widehat{Q}(t-i), \sum_{j=1}^{J_{D}} \mathcal{R}_{j}^{(D)}\left(\widehat{\alpha}_{j}^{(D)}(t), \widehat{D}(t-j)\right)+\widehat{\varepsilon}^{(D)}(t)\right] \\
& =\sum_{j=1}^{J_{D}} \widehat{\alpha}_{j}^{(D)}(t) \widehat{\operatorname{Cov}}[\widehat{Q}(t-i), \widehat{D}(t-j)] .
\end{aligned}
$$

The values of $\left\{\widehat{\operatorname{Cov}}[\widehat{Q}(-i), \widehat{D}(-j)] ; i, j=0,1,2, \ldots, \max \left\{J_{Q}, J_{D}\right\}-1\right\}$ can be typically obtained from the historical data to serve as the seed to initiate the computation in (D.27) and D.28). And $\widehat{\operatorname{Cov}}\left[\widehat{\varepsilon}^{(Q)}(t), \widehat{\varepsilon}^{(D)}(t)\right]$ can be obtained by plugging (D.26) and D.18) into (D.25).

\section{Appendix E. Generation of Bivariate Innovations}

For the readers' convenience, the copula-based algorithms in [90, 91] are provided here for the generation of the bivariate innovation process $\left(\widehat{\varepsilon}^{(Q)}(t), \widehat{\varepsilon}^{(D)}(t)\right)^{\top}$. The characteristics of $\left(\widehat{\varepsilon}^{(Q)}(t), \widehat{\varepsilon}^{(D)}(t)\right)^{\top}$ are estimated and give as: $\widehat{\mathrm{E}}\left[\widehat{\varepsilon}^{(Q)}(t)\right], \widehat{\mathrm{E}}\left[\widehat{\varepsilon}^{(D)}(t)\right], \widehat{\operatorname{Var}}\left[\widehat{\varepsilon}^{(Q)}(t)\right], \widehat{\operatorname{Var}}\left[\widehat{\varepsilon}^{(D)}(t)\right]$ 
and $\widehat{\operatorname{Cov}}\left[\widehat{\varepsilon}^{(Q)}(t), \widehat{\varepsilon}^{(D)}(t)\right]$. The marginal cumulative distribution functions (CDF) for $\widehat{\varepsilon}^{(Q)}(t)$ and $\left.\widehat{\varepsilon}^{(D)}(t)\right)^{\top}$ are specified as $F_{Q}$ and $F_{D}$, respectively. In this work, the CDF could be normal or generalized Poisson (GP) as discussed in Section 5.1.1, and herein we consider the case where at least one of $F_{Q}$ and $F_{D}$ follows GP.

In the notations below, the time index $t$ is omitted for clarity. Let $\mathbf{u}=\left(u^{(Q)}, u^{(D)}\right)^{\top}$ be a bivariate normal random variable with

$$
\mathrm{E}[\mathbf{u}]=\mathbf{0}, \quad \operatorname{Cov}[\mathbf{u}]=\left(\begin{array}{ll}
1 & \varphi \\
\varphi & 1
\end{array}\right) .
$$

Define $\widehat{\varepsilon}$ as

$$
\widehat{\varepsilon}=\left(\widehat{\varepsilon}^{(Q)}, \widehat{\varepsilon}^{(D)}\right)^{\top}=\left(F_{Q}^{-1}\left(\Phi\left(u^{(Q)}\right)\right), F_{D}^{-1}\left(\Phi\left(u^{(D)}\right)\right)\right)^{\top}
$$

The $\Phi$ denotes the CDF of stand normal distribution. The covariance between $\widehat{\varepsilon}^{(Q)}$ and $\widehat{\varepsilon}^{(D)}$ is given as:

$$
\operatorname{Cov}\left[\widehat{\varepsilon}^{(Q)}, \widehat{\varepsilon}^{(D)}\right]=\mathrm{E}\left[\widehat{\varepsilon}^{(Q)} \widehat{\varepsilon}^{(D)}\right]-\mathrm{E}\left[\widehat{\varepsilon}^{(Q)}\right] \mathrm{E}\left[\widehat{\varepsilon}^{(D)}\right]
$$

where

$$
\mathrm{E}\left[\widehat{\varepsilon}^{(Q)} \widehat{\varepsilon}^{(D)}\right]=\int_{-\infty}^{\infty} \int_{-\infty}^{\infty} F_{Q}^{-1}\left(\Phi\left(u^{(Q)}\right)\right) F_{D}^{-1}\left(\Phi\left(u^{(D)}\right)\right) \phi_{\varphi}\left(u^{(Q)}, u^{(D)}\right) d u^{(Q)} d u^{(D)} .
$$

In E.1), $\phi_{\varphi}\left(u^{(Q)}, u^{(D)}\right)$ denotes the probability density function of $\mathbf{u}$. Obviously, the value of $\mathrm{E}\left[\widehat{\varepsilon}^{(Q)} \widehat{\varepsilon}^{(D)}\right]$ depends on the choice of $\varphi$. Hence at this point, the problem of generating $\widehat{\varepsilon}$ with given characteristics turns into the covariance matching problem: Find the value of $\varphi$ such that

$$
\mathrm{E}\left[\widehat{\varepsilon}^{(Q)} \widehat{\varepsilon}^{(D)}\right]=\widehat{\operatorname{Cov}}\left[\widehat{\varepsilon}^{(Q)}, \widehat{\varepsilon}^{(D)}\right]+\widehat{\mathrm{E}}\left[\widehat{\varepsilon}^{(Q)}\right] \widehat{\mathrm{E}}\left[\widehat{\varepsilon}^{(D)}\right] .
$$

To solve the covariance matching problem in $(\mathrm{E} .2)$, the integral of $E\left[\widehat{\varepsilon}^{(Q)} \widehat{\varepsilon}^{(D)}\right]$ in E.1) is first transformed into a sum of terms, and then a numerical algorithm is employed to find $\widehat{\varphi}$ that solves (E.2) approximately 91] and [90].

Given the $F_{Q}, F_{D}$ and $\widehat{\operatorname{Cov}}\left[\widehat{\varepsilon}^{(Q)}, \widehat{\varepsilon}^{(D)}\right]$, the algorithm for generating samples of bivariate random variables $\left\{\widehat{\varepsilon}_{i} ; i=1,2, \ldots, N\right\}$ are summarized as follow:

Step 1 : Use the the formula (19) in [90] if both $\widehat{\varepsilon}^{(Q)}$ and $\widehat{\varepsilon}^{(D)}$ follow GP distribution, or (11) in [91] if one of them follows normal distribution, to find $\widehat{\varphi}$ that solves $($ E.2 via numerical search (e.g. the Newton-Raphson method).

Step 2 : Generate independent bivariate standard normal variables $\left\{\mathbf{u}_{i}=\left(u_{i}^{(Q)}, u_{i}^{(D)}\right)^{\top}, i=\right.$ $1,2, \ldots, N\}$, where each $\mathbf{u}_{i}$ satisfies:

$$
\mathrm{E}\left[\mathbf{u}_{i}\right]=\mathbf{0}, \quad \operatorname{Cov}\left[\mathbf{u}_{i}\right]=\left(\begin{array}{ll}
1 & \widehat{\varphi} \\
\widehat{\varphi} & 1
\end{array}\right)
$$


Many softwares (e.g. Matlab, R) provide efficient algorithms to complete this step.

Step 3 : For each $i$, obtain $\widehat{\varepsilon}_{i}=\left(\widehat{\varepsilon}_{i}^{(Q)}, \widehat{\varepsilon}_{i}^{(D)}\right)^{\top}$ by

$$
\widehat{\boldsymbol{\varepsilon}}_{i}^{(Q)}=F_{Q}^{-1}\left(\Phi\left(z_{i}^{(Q)}\right)\right), \quad \widehat{\varepsilon}^{(D)}=F_{D}^{-1}\left(\Phi\left(z_{i}^{(D)}\right)\right) .
$$

\section{Appendix F. Two-Stage Procedure for Sample Size Determination}

For a candidate release plan $\mathbf{x}$, how to determine the number of Monte Carlo simulation (MCS) replications $R$ needed to obtain high-quality estimates of the performance metrics: the expected total cost $\mathrm{E}[T C(\mathbf{x})]$ and the fill rate $\mathrm{E}[F R(\mathbf{x})]$ ? Denote a performance metric as $M(\mathbf{x})$ in general, where $M(\mathbf{x})$ can be $T C(\mathbf{x})$ or $F R(\mathbf{x})$. In this work, the two-stage procedure [41, 54] is implemented to determine the value of $R$ so that a desired precision can be achieved for $\widehat{\mathrm{E}}[M(\mathbf{x})]$, that is,

$$
\frac{\widehat{\operatorname{Std}}[\widehat{\mathrm{E}}[M(\mathbf{x})]]}{\widehat{\mathrm{E}}[M(\mathbf{x})]}<\gamma \%
$$

where $\widehat{\operatorname{Std}}[\widehat{\mathrm{E}}[M(\mathbf{x})]]$ is the estimated standard deviation of $\widehat{\mathrm{E}}[M(\mathbf{x})]$, and $\gamma \%$ the target precision level. At the first stage, an initial number of replications $R_{0}$ are generated. Denote $\widehat{\mathrm{E}}_{0}[M(\mathbf{x})]$ as the estimated mean of $M(\mathbf{x})$ and $\widehat{\operatorname{Std}}_{0}\left[\widehat{\mathrm{E}}_{0}[M(\mathbf{x})]\right]$ as the estimated standard deviation of $\widehat{\mathrm{E}}_{0}[M(\mathbf{x})]$ from the $R_{0}$ replications. Then the number of replications $R$ that is likely to achieve the target precision level $\gamma \%$ is estimated as

$$
R=\left\lceil\widehat{\operatorname{Std}}_{0}\left[\widehat{\mathrm{E}}_{0}[M(\mathbf{x})]\right] /\left(\widehat{\mathrm{E}}_{0}[M(\mathbf{x})] \times \gamma \%\right)\right\rceil
$$

At the second stage, $R-R_{0}$ additional replications are generated and the data collected at both stages are used to estimate the performance metrics.

\section{Appendix G. Simulating the Demand Process}

The demand process $\mathfrak{D}(t)$ is written as:

$$
\mathfrak{D}(t)=\mathrm{E}[\mathfrak{D}(t)]+v(t), \quad t=1,2, \ldots, H
$$

where $\mathrm{E}[\mathfrak{D}(t)]$ is pre-given, and $v(t)$ is a stochastic process representing the deviation of $\mathfrak{D}(t)$ from its mean $\mathrm{E}[\mathfrak{D}(t)]$. Denote the demand realization times as

$$
\left\{\tau_{1}, \tau_{2}, \ldots, \tau_{U}\right\} ; \quad 1 \leq \tau_{1}<\tau_{2}<\ldots<\tau_{U} \leq H
$$


with $U$ being the number of demand realizations over the planning horizon. $\mathrm{E}[\mathfrak{D}(t)]$ and $v(t)$ are set as 0 at the time points when no demand is realized, that is

$$
\mathrm{E}[\mathfrak{D}(t)]=0, v(t)=0, t \notin\left\{\tau_{1}, \tau_{2}, \ldots, \tau_{U}\right\} .
$$

In this work, $v\left(\tau_{i}\right)$ is modeled by a AR-GARCH process [100, 101], which is widely used in modeling and forecasting demand [94 97]. The AR-GARCH model is given as

$$
\begin{gathered}
v\left(\tau_{i}\right)=\xi v\left(\tau_{i-1}\right)+\epsilon\left(\tau_{i}\right) \sigma\left(\tau_{i}\right) \\
\sigma\left(\tau_{i}\right)^{2}=\phi_{0}+\phi_{1}\left(\epsilon\left(\tau_{i-1}\right) \sigma\left(\tau_{i-1}\right)\right)^{2}+\psi \sigma\left(\tau_{i-1}\right)^{2}
\end{gathered}
$$

where $\epsilon\left(\tau_{i}\right)$ is Gaussian white noise with unit variance, and $\xi, \phi_{0}, \phi_{1}$ and $\psi$ are given model parameters.

\section{Appendix H. Behavior of the NSGA-II Algorithm}

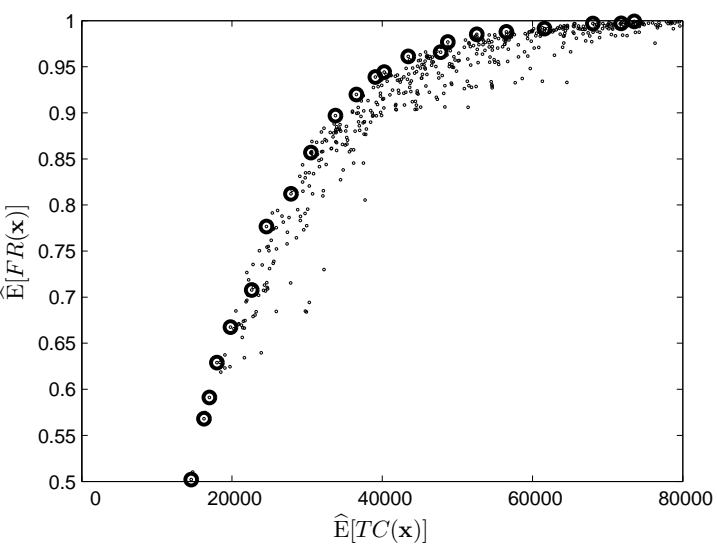

(a) Scenario 1

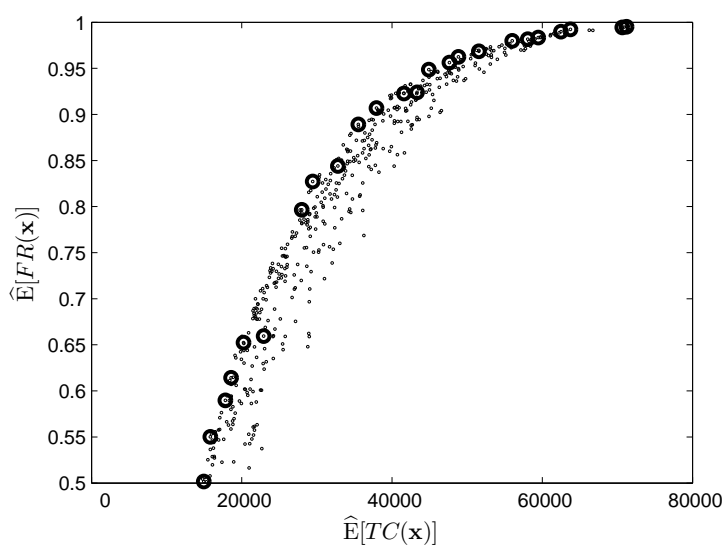

(c) Scenario 3

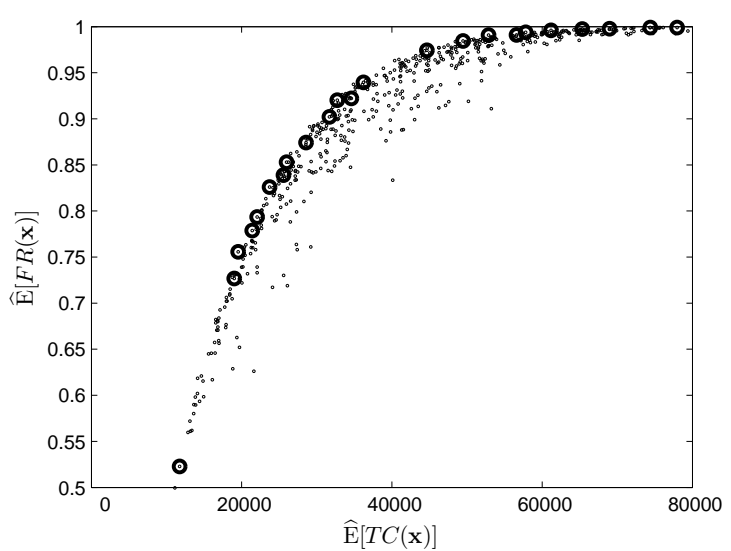

(b) Scenario 2

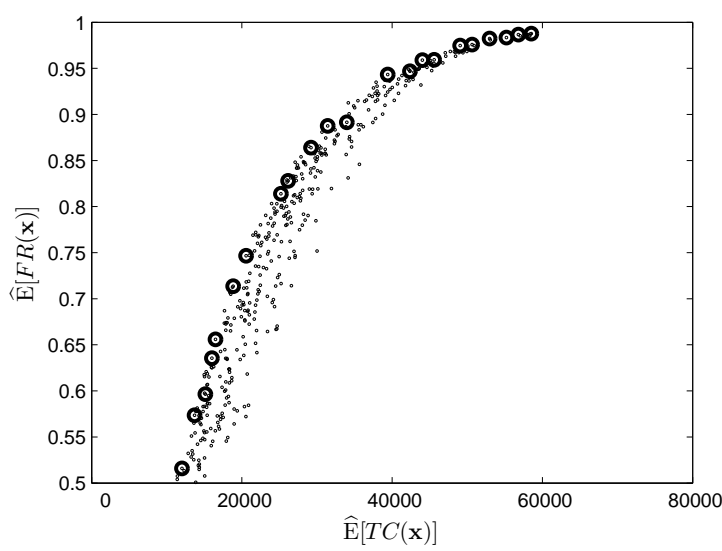

(d) Scenario 4

Figure H.11: "o" highlights non-dominated solutions found by the NSGA-II algorithm. "." corresponds to all candidate plans visited by the NSGA-II algorithm. 
Figure H.11 displays for each demand scenario all the candidate plans visited by the NSGA-II algorithm. Each plan is denoted as a dot in the two-dimensional performancemetric space: the expected demand fulfill rate $\mathrm{E}[F R(\mathbf{x})]$ versus the expected total cost $\mathrm{E}[T C(\mathbf{x})]$. The plans included in the Pareto frontier by the algorithm are depicted as circled dots. 


\section{References}

[1] Missbauer H, Uzsoy R. Optimization models of production planning problems. In: Kempf KG, Keskinocak P, Uzsoy R, editors. Planning Production and Inventories in the Extended Enterprise: A State of the Art Handbook. New York: Springer; 2011, p. 437 - 507.

[2] Heizer JH, Render B. Operations management. Upper Saddle River, NJ: Pearson Prentice Hall; 2008.

[3] Kempf KG, Uzsoy R, Keskinocak P. Planning production and inventories in the extended enterprise: a state of the art handbook. Springer; 2010.

[4] Anthony RN. Planning and control systems: a framework for analysis. Harvard University Press; 1965.

[5] Bitran GR, Tirupati D. Hierarchical production planning. Handbooks in Operations Research and Management Science 1993;4:523-68.

[6] Buzacott JA, Shanthikumar JG. Stochastic models of manufacturing systems; vol. 4. Prentice Hall Englewood Cliffs, NJ; 1993.

[7] Hopp WJ, Spearman ML. Factory physics: foundations of manufacturing management. Homewood: Irwin; 2001.

[8] Atherton RW, Dayhoff JE. Signature analysis: simulation of inventory, cycle time, and throughput trade-offs in wafer fabrication. IEEE Transactions on Components, Hybrids, and Manufacturing Technology 1986;9(4):498-507.

[9] Ankenman BE, Bekki JM, Fowler JW, Mackulak GT, Nelson BL, Yang F. Simulation in production planning: an overview with emphasis on recent developments in cycle time estimation. In: Kempf KG, Keskinocak P, Uzsoy R, editors. Planning Production and Inventories in the Extended Enterprise: A State of the Art Handbook. Springer; 2011, p. 565-91.

[10] Johnson LA, Montgomery DC. Operations research in production planning, scheduling, and inventory control. New York: Wiley; 1974.

[11] Voß S, Woodruff DL. Introduction to computational optimization models for production planning in a supply chain; vol. 240. Springer; 2006.

[12] Pochet Y, Wolsey LA. Production planning by mixed integer programming. Springer; 2006.

[13] Hackman S. Production economics: integrating the microeconomic and engineering. Springer-Verlag, Heidelberg; 2008.

[14] Missbauer H. Order release planning with clearing functions: a queueing-theoretical analysis of the clearing function concept. International Journal of Production Economics 2011;131:399-406.

[15] Orlicky J. Material requirements planning: the new way of life in production and inventory management. McGraw-Hill, New York; 1975.

[16] Vollmann TE, Berry WL, Whybark DC, Jacobs FR. Manufacturing planning and control for supply chain management. McGraw-Hill/Irwin New York; 2005.

[17] Baker KR. Requirements planning. Handbooks in operations research and management science 1993;4:571-627.

[18] Leachman RC, Benson RF, Liu C, Raar DJ. Impress: an automated production planning and delivery quotation system at harris corporation semiconductor sector. Interfaces 1996;26(1):6-37.

[19] Fordyce K, Wang CT, Chang CH, Degbotse A, Denton B, Lyon P, et al. The ongoing challenge: creating an enterprise-wide detailed supply chain plan for semiconductor and package operations. In: Planning Production and Inventories in the Extended Enterprise. Springer; 2011, p. 313-87.

[20] Peters R, Boskma K, Kupper H. Stochastic programming in production planning: a case with nonesimple recourse. Statistica Neerlandica 1977;31(3):113-26.

[21] Escudero LF, Kamesam PV, King AJ, Wets RJ. Production planning via scenario modelling. Annals of Operations Research 1993;43(6):309-35. 
[22] Sen S, Higle JL. An introductory tutorial on stochastic linear programming models. Interfaces 1999;29(2):33-61.

[23] Higle JL, Kempf KG. Production planning under supply and demand uncertainty: A stochastic programming approach. In: Infanger G, editor. Stochastic Programming: The State of the Art. Berlin: Springer; 2010, p. $297-315$.

[24] Bertsimas D, Sim M. The price of robustness. Operations Research 2004;52(1):35-53.

[25] Bertsimas D, Thiele A. A robust optimization approach to inventory theory. Operations Research 2006;54(1):150-68.

[26] Charnes A, Cooper WW. Chance constrained programming. Management Science 1959;6(1):73-9.

[27] Prékopa A. Stochastic programming; vol. 324. Springer Science \& Business Media; 2013.

[28] Aouam T, Uzsoy R. Chance-constraint-based heuristics for production planning in the face of stochastic demand and workload-dependent lead times. In: Decision Policies for Production Networks. Springer; 2012, p. 173-208.

[29] Aouam T, Uzsoy R. Zero-order production planning models with stochastic demand and workloaddependent lead times. International Journal of Production Research 2014;(ahead-of-print):1-19.

[30] Pahl J, Voß S, Woodruff DL. Production planning with load dependent lead times. 4OR 2005;3(4):257302.

[31] Pahl J, Voß S, Woodruff DL. Production planning with load dependent lead times: an update of research. Annals of Operations Research 2007;153(1):297-345.

[32] Srinivasan A, Carey M, Morton TE. Resource pricing and aggregate scheduling in manufacturing systems. Tech. Rep.; Carnegie Mellon University, Tepper School of Business; 1988.

[33] Asmundsson JM, Rardin RL, Uzsoy R. Production planning models for resources subject to congestion. Naval Research Logistics 2009;56:142-57.

[34] Selçuk B. FJC, De Kok AG. Work-in-process clearing in supply chain operations planning. IIE Transactions 2008;40(3):206-20.

[35] Missbauer H. Models of the transient behaviour of production units to optimize the aggregate material flow. International Journal of Production Economics 2009;118(2):387-97.

[36] Asmundsson JM, Rardin RL, Uzsoy R. Tractable nonlinear production planning models for semiconductor wafer fabrication facilities. IEEE Transactions on Semiconductor Manufacturing 2006;19(1):95111.

[37] Kacar NB, Irdem DF, Uzsoy R. An experimental comparison of production planning using clearing functions and iterative linear programming-simulation algorithms. IEEE Transactions on Semiconductor Manufacturing 2012;25(1):104-17.

[38] Kacar NB, Monch L. aUR. Planning wafer starts using nonlinear clearing dunctions: a large-scale experiment. IEEE Transactions on Semiconductor Manufacturing 2013;26(4):602-12.

[39] Hasan CN, Spearman ML. Optimal material release times in stochastic production environments. International Journal of Production Research 1999;37(6):1201-16.

[40] Homem-De-Mello T, Shapiro A, Spearman ML. Finding optimal material release times using simulation-based optimization. Management Science 1999;45(1):86-102.

[41] Liu J, Li C, Yang F, Wan H, Uzsoy R. Production planning for semiconductor manufacturing via simulation optimization. In: Jain S, Creasey RR, Himmelspach J, White KP, Fu M, editors. Proceedings of the Winter Simulation Conference. New York; 2011, p. 3617-27.

[42] Kacar NB, Uzsoy R. Estimating clearing functions for production resources using simulation optimization. Automation Science and Engineering, IEEE Transactions on 2015;12(2):539-52.

[43] Gansterer M, Almeder C, Hartl RF. Simulation-based optimization methods for setting production planning parameters. International Journal of Production Economics 2014;151:206-13.

[44] Albey E, Uzsoy R. Lead time modeling in production planning. In: Proceedings of the Winter 
Simulation Conference. Huntington Beach, CA; 2015,.

[45] Hung YF, Leachman RC. A production planning methodology for semiconductor manufacturing based on iterative simulation and linear programming calculations. IEEE Transactions on Semiconductor Manufacturing 1996;9(2):257-69.

[46] Byrne MD, Bakir MA. Production planning using a hybrid simulationanalytical approach. International Journal of Production Economics 1999;59(1):305-11.

[47] Kim B, Kim S. Extended model for a hybrid production planning approach. International Journal of Production Economics 2001;73(2):165-73.

[48] Byrne MD, Hossain MM. Production planning: an improved hybrid approach. International Journal of Production Economics 2005;93-94:225-9.

[49] Bang JY, Kim YD. Hierarchical production planning for semiconductor wafer fabrication based on linear programming and discrete-event simulation. IEEE Transactions on Automation Science and Engineering 2010;7(2):326-36.

[50] Irdem DF, Kacar NB, Uzsoy R. An exploratory analysis of two iterative linear programmingsimulation approaches for production planning. IEEE Transactions on Semiconductor Manufacturing 2010;23(3):442-55.

[51] Orcun S, Uzsoy R, Kempf KG. An integrated production planning model with load-dependent leadtimes and safety stocks. Computers \& Chemical Engineering 2009;33(12):2159-63.

[52] Kacar NB, Uzsoy R. A comparison of multiple linear regression approaches for fitting clearing functions to empirical data. International Journal of Production Research 2014;52(11):3164-84.

[53] Ankenman B, Nelson BL, Staum J. Stochastic kriging for simulation metamodeling. Operations Research 2010;58(2):371-82.

[54] Yang F, Liu J. Simulation-based transfer function modeling for transient analysis of general queueing systems. European Journal of Operational Research 2012;223:150-66.

[55] Gerhardt I, Nelson BL. Transforming renewal processes for simulation of nonstationary arrival processes. INFORMS Journal on Computing 2009;21(4):630-40.

[56] Nelson BL, Gerhardt I. Modelling and simulating non-stationary arrival processes to facilitate analysis. Journal of Simulation 2011;5(1):3-8.

[57] Box GE, Jenkins GM, Reinsel GC. Time series analysis: forecasting and control. John Wiley \& Sons; 2013.

[58] del Castillo J, Pérez-Casany M. Overdispersed and underdispersed Poisson generalizations. Journal of Statistical Planning and Inference 2005;134(2):486-500.

[59] Jung RC, Tremayne AR. Convolution-closed models for count time series with applications. Journal of Time Series Analysis 2011;32(3):268-80.

[60] Jung RC, Tremayne AR. Useful models for time series of counts or simply wrong ones? AStA Advances in Statistical Analysis 2011;95(1):59-91.

[61] Weiß CH. Integer-valued autoregressive models for counts showing underdispersion. Journal of Applied Statistics 2013;40(9):1931-48.

[62] Du J, Li Y. The integer-value autoregressive (inar(p)) model. Journal of Time Series Analysis 1991;12(2):129-42.

[63] Fokianos K. Count time series models. Handbook in Statistics: Time Series Methods and Applications 2012;30:315-48.

[64] McKenzie E. Discrete variate time series. Handbook of Statistics 2003;21:573-606.

[65] Jung RC, Kukuk M, Liesenfeld R. Time series of count data: modeling, estimation and diagnostics. Computational Statistics \& Data Analysis 2006;51(4):2350-64.

[66] Grunwald GK, Hyndman RJ, Tedesco L, Tweedie RL. Theory \& methods: non-gaussian conditional linear AR(1) models. Australian \& New Zealand Journal of Statistics 2000;42(4):479-95. 
[67] Kachour M, Truquet L. A p-order signed integer-valued autoregressive (SINAR(p)) model. Journal of Time Series Analysis 2011;32(3):223-36.

[68] Kachour M, Yao J. First-order rounded integer-valued autoregressive (RINAR(1)) process. Journal of Time Series Analysis 2009;30(4):417-48.

[69] Alzaid AA, Al-Osh M. An integer-valued pth-order autoregressive structure (INAR(p)) process. Journal of Applied Probability 1990;:314-24.

[70] Weiß CH. Thinning operations for modeling time series of counts-a survey. AStA Advances in Statistical Analysis 2008;92(3):319-41.

[71] Joe H. Time series models with univariate margins in the convolution-closed infinitely divisible class. Journal of Applied Probability 1996;:664-77.

[72] Davis RA, Dunsmuir WTM, Streett SB. Observation-driven models for Poisson counts. Biometrika 2003;90(4):777-90.

[73] Zeger SL. A regression model for time series of counts. Biometrika 1988;75(4):621-9.

[74] Zeger SL, Qaqish B. Markov regression models for time series: a quasi-likelihood approach. Biometrics $1988 ;: 1019-31$.

[75] Zhu F. Modeling overdispersed or underdispersed count data with generalized Poisson integer-valued GARCH models. Journal of Mathematical Analysis and Applications 2012;389(1):58-71.

[76] Kim H, Park Y. A non-stationary integer-valued autoregressive model. Statistical Papers 2008;49(3):485-502.

[77] Rao TS. The fitting of non-stationary time-series models with time-dependent parameters. Journal of the Royal Statistical Society Series B (Methodological) 1970;32(2):312-22.

[78] Brännäs K. Explanatory variables in the AR(1) count data model. Umeå Economic Studies 1995;381.

[79] Enciso-Mora V. NP, Rao TS. Integer valued AR processes with explanatory variables. Sankhyā: The Indian Journal of Statistics, Series B 2009;:248-63.

[80] Consul PC. Generalized Poisson distributions: properties and applications. New York and Basel: M. Dekker; 1989.

[81] Efron B. Double exponential families and their use in generalized linear regression. Journal of the American Statistical Association 1986;81(395):709-21.

[82] Sellers K. F. BS, Shmueli G. The COM-Poisson model for count data: a survey of methods and applications. Applied Stochastic Models in Business and Industry 2012;28(2):104-16.

[83] Steutel FW, Van Harn K. Discrete analogues of self-decomposability and stability. The Annals of Probability 1979;7(5):893-9.

[84] Chesneau C, Kachour M. A parametric study for the first-order signed integer-valued autoregressive process. Journal of Statistical Theory and Practice 2012;6(4):760-82.

[85] Wilk MB, Gnanadesikan R. Probability plotting methods for the analysis for the analysis of data. Biometrika 1968;55(1):1-17.

[86] Massey Jr FJ. The Kolmogorov-Smirnov test for goodness of fit. Journal of the American Statistical Association 1951;46(253):68-78.

[87] Shapiro SS, Wilk MB. An analysis of variance test for normality (complete samples). Biometrika 1965;:591-611.

[88] Brockwell PJ, Davis RA. Introduction to time series and forecasting; vol. 1. Taylor \& Francis; 2002.

[89] Ripley BD. Stochastic simulation; vol. 316. John Wiley \& Sons; 2009.

[90] Avramidis AN, Channouf N, L'Ecuyer P. Efficient correlation matching for fitting discrete multivariate distributions with arbitrary marginals and normal-copula dependence. INFORMS Journal on Computing 2009;21(1):88-106.

[91] Channouf N, L'Ecuyer P. Fitting a normal copula for a multivariate distribution with both discrete and continuous marginals. In: Proceedings of the Winter Simulation Conference. Winter Simulation 
Conference; 2009, p. 352-8.

[92] Kayton D, Teyner T, Schwartz C, Uzsoy R. Focusing maintenance improvement efforts in a wafer fabrication facility operating under theory of constraints. Production and Inventory Management 1997;38:51-7.

[93] Liu J. Simulation-based transfer function modeling approach for responsive production planning. Ph.D. thesis; West Virginia University; 2011.

[94] Garcia R. C. CJVAM, Garcia JBC. A GARCH forecasting model to predict day-ahead electricity prices. Power Systems, IEEE Transactions on 2005;20(2):867-74.

[95] Song H, Li G. Tourism demand modelling and forecastinga review of recent research. Tourism Management 2008;29(2):203-20.

[96] Taylor JW, Buizza R. A comparison of temperature density forecasts from GARCH and atmospheric models. Journal of Forecasting 2004;23(5):337-55.

[97] Taylor J. W. MPE, Buizza R. Wind power density forecasting using ensemble predictions and time series models. IEEE Transactions on Energy Conversion 2009;24(3):775-82.

[98] Deb K. Multi-objective optimization using evolutionary algorithms; vol. 16. John Wiley \& Sons; 2001.

[99] Brockwell PJ, Davis RA. Time series: theory and methods. Springer; 2009.

[100] Bollerslev T. Generalized autoregressive conditional heteroskedasticity. Journal of Econometrics 1986;31(3):307-27.

[101] Engle RF. Autoregressive conditional heteroscedasticity with estimates of the variance of United Kingdom inflation. Econometrica: Journal of the Econometric Society 1982;:987-1007. 